\title{
Heel Height as an Etiology of Hallux Abductus Valgus Development: An electromagnetic Static and Dynamic First Metatarsophalangeal Joint Study
}

\author{
Rubén Sánchez-Gómez ${ }^{1,2} * \mathbb{*}$, Ricardo Becerro de Bengoa-Vallejo ${ }^{2} \oplus$, \\ Marta Elena Losa-Iglesias ${ }^{3}$ D , César Calvo-Lobo ${ }^{4}$, Carlos Romero-Morales ${ }^{1}$, \\ Eva María Martínez-Jiménez ${ }^{2}$ (D) Patricia Palomo-López ${ }^{5}$ iD and Daniel López-López ${ }^{6}$ (D) \\ 1 Faculty of Sports Sciences, Universidad Europea de Madrid, 28670 Villaviciosa de Odón, Madrid, Spain; \\ carlos.romero@universidadeuropea.es \\ 2 Facultad de Enfermería, Fisioterapia y Podología, Universidad Complutense de Madrid, 28040 Madrid, \\ Spain; ribebeva@ucm.es (R.B.d.B.-V.); eva.hache2@hotmail.com (E.M.M.-J.) \\ 3 Faculty of Health Sciences, Universidad Rey Juan Carlos, 28922 Alcorcón, Spain; marta.losa@urjc.es \\ 4 Nursing and Physical Therapy Department, Institute of Biomedicine (IBIOMED), Faculty of Health Sciences, \\ Universidad de León, Ponferrada, 24071 León, Spain; ccall@unileon.es \\ 5 University Center of Plasencia, Universidad de Extremadura, 10600 Plasencia, Spain; patibiom@unex.es \\ 6 Research, Health and Podiatry Unit, Department of Health Sciences, Faculty of Nursing and Podiatry, \\ Universidade da Coruña, 15403 Ferrol, Spain; daniellopez@udc.es \\ * Correspondence: rusanc02@ucm.es
}

Received: 19 December 2018; Accepted: 13 March 2019; Published: 16 March 2019

check for updates

\begin{abstract}
Background: Hallux abductus valgus (HAV) is a forefoot condition produced by extrinsic and intrinsic factors. Shoes with a high heel height and a typical narrow tip toe box can induce deviations in both the proximal phalanx of the hallux (PPH) and the first metatarsal (IMTT) bones. Nevertheless, the isolated role of heel height remains unclear in the development of HAV pathology. Objectives: The goal was to determine if the heel height increase of shoes without a narrow box toe could augment the PPH and IMTT deviation in frontal, sagittal, and transverse planes toward the first metatarsophalangeal joint (MPJ) and the first metatarsocuneiform joint (MCJ), respectively, during static and dynamic conditions in relation to precursor movements of HAV. Methods: Women with an average age of $25.10 \pm 4.67$ years were recruited in this cross-sectional study to assess the three planes of motion of PPH and IMTT while wearing high heels with heights at 3, 6, $9 \mathrm{~cm}$ and unshod conditions via sandals. The measurements used an electromagnetic goniometer device with sensors placed on medial aspects of the PPH and IMTT bones under static and dynamic conditions. Results: Wearing shoes with a $6 \mathrm{~cm}$ heel in dynamic condition may increase the PPH valgus and abduction deviation from $3.15 \pm 0.10^{\circ}$ to $3.46 \pm 0.05^{\circ}(p<0.05)$ and from $1.35 \pm 0.28^{\circ}$ to $1.69 \pm 0.30^{\circ}(p<0.001)$, respectively. In addition, a PPH abduction increase from $1.01 \pm 0.36^{\circ}$ to $1.31 \pm 0.46^{\circ}(p<0.05)$ after wearing shoes with a $6 \mathrm{~cm}$ heel height was observed under static conditions. Conclusions: Wearing shoes with a heel height of $6 \mathrm{~cm}$ without a narrow box toe interference may produce PPH abduction and valgus deviations related to HAV formation.
\end{abstract}

Keywords: hallux abductus valgus; high heel; proximal phalanx of the hallux; abduction; valgus

\section{Introduction}

Hallux Abductus Valgus (HAV) is a pathological subluxation of the first metatarsophalangeal joint (IMPJ) with lateral deviation of the proximal phalanx of the hallux bone (PPH) toward abduction and valgus direction in both the transverse and frontal planes of motion, respectively. There is also 
deviation of the first metatarsocuneiform joint (IMCJ) through the first metatarsal bone (IMTT) toward adduction and valgus direction in both transverse and frontal planes of motion, respectively. There are occasionally bony enlargements of the first metatarsal head (also called a "bunion") [1-3]. The IMPJ bears $80 \%$ of the body load without help of any structure in heel-off phase, and this makes it a more sensitive joint to biomechanics deformities such HAV [1].

The etiology of HAV can have multiple origins, and there are intrinsic and extrinsic factors in play [4-9]. Intrinsic factors include hyperpronation [5,10], soft tissue weakness [1], and hyperlaxity with medial longitudinal arch collapse [11,12]; Windlass mechanism failure [7], first ray hypermobility [8], and female sex [13-15] have been linked with HAV growth. As extrinsic factors, the use of high-heel shoes has also been detected as a possible cause of HAV development: The typical high-heeled shoe for women can lead to bad body repercussions and be detrimental to bone mineralization [16], rear foot instability [17], body mass center changes [18], biomechanics gait changes [19,20], and general damage health [21].

Some authors have reported increases in concentrate load under the forefoot with high heeled shoes [22-25], and this condition can predispose the subject to HAV development [26]. A few studies have speculated that the current narrow box of high-heeled shoes are not the only cause of HAV because isolated high-heel shoes might cause weight to be placed on the forefoot, and this may overstretch the toes and lead to the development of splayfoot $[1,27,28]$. However, this has not been reported in the literature.

The 2D [29] and 3D kinematic movements of PPH and I MTT bone deviation during gait in subjects with and without HAV has shown the relationship between the rearfoot and midfoot eversion with respect to the first ray hypermobility and the presence on HAV [10,30]; other groups have studied the kinematic effects of improvements in taping in subjects with HAV during gait [31] or the negative effects of HAV surgery to normal ambulation [32]. Other work studied the kinematics effects on IMPJ using foot orthoses that incorporated forefoot and rearfoot posting - the results showed no negative effects on mobility [33]. One prior study [34] identified kinetic evidence of wearing $5 \mathrm{~cm}$-heeled shoes during gait can lead to HAV development and an increase in hallux dorsiflexion in the final push-off phase; however, no report has described the transverse or frontal plane motion of the PPH or IMTT.

Therefore, the goal of this study is to determine how the heel height affects PPH and IMTT bone deviations either in the three planes of motion in static conditions and during the dorsiflexion of the IMPJ sequence (push-off phase of the dynamic condition) regardless of toe box of the shoe. The results can show if there is some movement related to HAV deviation that is characterized by $\mathrm{PPH}$ in abduction (away to medial body line) in transverse plane and valgus deviation in frontal plane toward the IMPJ [2] and/or the adduction deviation of the IMTT (toward medial body line) regarding the second MTT in transverse plane and the presence of the valgus in the frontal plane toward the IMCJ [2].

\section{Materials and Methods}

\subsection{Subjects}

The institutional review board at Rey Juan Carlos University approved the study. All subjects signed the informed consent form prior to beginning this study. The following inclusion criteria were required to participate in this study [35]: (1st) to have at least $10^{\circ}$ dorsiflexion of the ankle after complete knee fully dorsiflexion; (2nd) to have at least $30^{\circ}$ motion in the functional subtalar joint; (3rd) to have at least $15^{\circ}$ of motion along the mid-tarsal joint longitudinal axis; (4th) to reach at least $8 \mathrm{~mm}$ of non-weight bearing motion of the first ray; (5th) older than 18 years and younger than 38 years; (6th) no lower limb pathology or chronic condition at the time of recruitment and measurement; and (7th) prior experience of the use of shoes with high heel height during at least the prior year [36]. The exclusion criteria included established hallux valgus; prior history of foot and lower limb traumas, fractures, or surgeries; and congenital deformities or existing diagnoses of neurological, inflammatory, 
metabolic or vascular diseases. Subjects were also excluded if they showed less than $40^{\circ}$ passive dorsiflexion of the first MPJ (as a non-weight bearing technique previously described according to Buell et al. [37]). This range has been reported to be the IMPJ dorsiflexion range used during normal propulsion [38,39] and indicates joint structural limitations. In addition, male sex was an exclusion criterion because they rarely wear high-heeled shoes.

\subsection{Sample Size}

The sample size was calculated with software from Unidad de Epidemiología Clínica y Bioestadística. Complexo Hospitalario Universitario de A Coruña. Universidade A Coruña (www.fisterra.com) to detect a significant difference in the deviation of the axes of IMTT and PPH during static and dynamic test wearing high heels versus barefoot similar in another study with 15 recruited subjects [34] where hallux dorsiflexion decreased while wearing high heels from $26.6 \pm 2.33^{\circ}$ to $22.5 \pm 1.62^{\circ}$ in barefoot condition with $80 \%$ statistical power. Here, $\beta=20 \%$ with a $95 \%$ interval of confidence and $\alpha=0.05$ using a 2-tailed test. Thus, at least 61 participants were required. Furthermore, assuming a loss to follow up rate of $15 \%$, at least 71 participants were included in the study. However, recruited 80 subjects.

\subsection{Instrumentation; Assessment of the IMTT and PPH}

Three-dimensional degrees of the angular deviation of the IMTT bone movement; dorsiflexion/plantar flexion, adduction/abduction, and inversion/eversion at the first MCJ; dorsiflexion/plantar flexion, adduction/abduction, and inversion/eversion of PPH bone; and dorsiflexion/plantarflexion, adduction/abduction and inversion/eversion at the first MPJ, with and without wearing high-heeled shoes were studied during static and push-off phases using a 6Space Fastrak $^{\circledR}$ (Polhemus, Inc., Colchester, VT, USA) (Figure 1). This system had a $120 \mathrm{~Hz}$ sampling rate and a excellent validity and reliability $(r>0.99)$ [38] with ICC $=0.88-0.99$ and $0.95-0.99$ (SEM $=0.7-0.8 \mathrm{~mm})$, respectively [40], according to previous studies; the equipment contained two sensors that send an electromagnetic field with $6^{\circ}$ of freedom relative to the electromagnetic emitter. A 30-foot serial cable connected the sensors and emitter to a receiver module that collected, filtered, and managed all the signal's information.

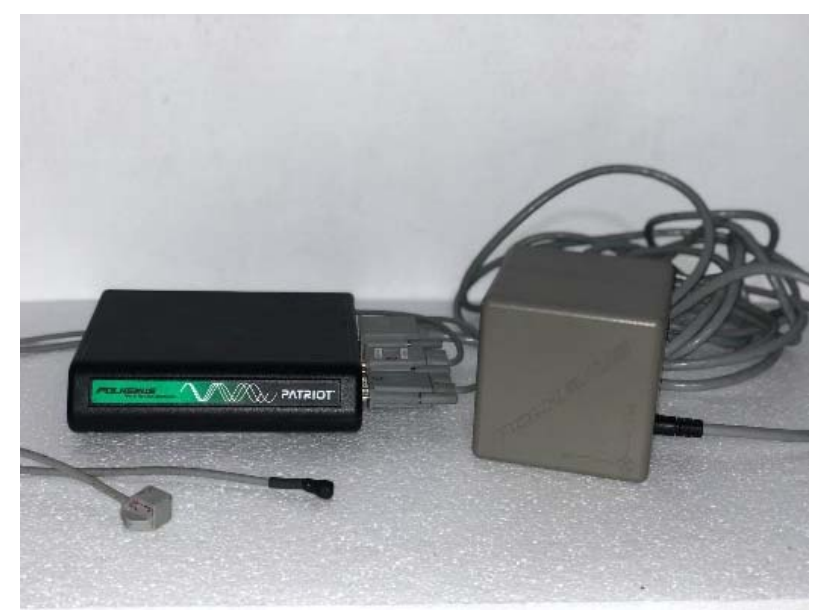

Figure 1. Polhemus device. From left to the right: sensors, receiver module, emitter module.

The signal from each sensor was captured by the receiver module. It was digitally transformed by the software to generate spatial orientation data from each sensor. The system showed a 8-mm static accuracy relative to the sensor position and $0.15^{\circ}$ regarding sensor orientation; the error was $1.6 \%[38,41]$. The relation between range vs. resolution in orientation aspect was $0.3 \mathrm{~m}$ to $1.16 \mathrm{~m}$; in addition, the relation between range vs. orientation aspect was 0.3 to $0.0038^{\circ}$. 
In this study, the electromagnetic emitter was $96 \mathrm{~cm}$ high at the midway point on a 6-m raised walkway. There were no metallic elements near the electromagnetic device or in the subject's walking path to avoid possible interference [42]. One of the sensors was placed at the medial aspect of the head of the IMTT bone (Figure 2), and the second one was located along the medial aspect of the PPH (Figure 3) on the right foot; the Polhemus Fastrack ${ }^{\circledR}$ (Polhemus, Inc., Colchester, VT, USA) did not measure angles but did measure bone displacements. Thus, we assessed the mobility of the PPH regardless of IMTT mobility to determine which areas were affected by the high heels. Both sensors were attached with tape and secured to the skin with an auto-adhesive bandage (Figure 4); this medial location was selected according to a protocol devised by Welsh et al. [43] to minimal overlying soft tissue of extensor hallucis longus tendon's excursion [41,44] All cables were fixed via straps to the thighs with a belt.

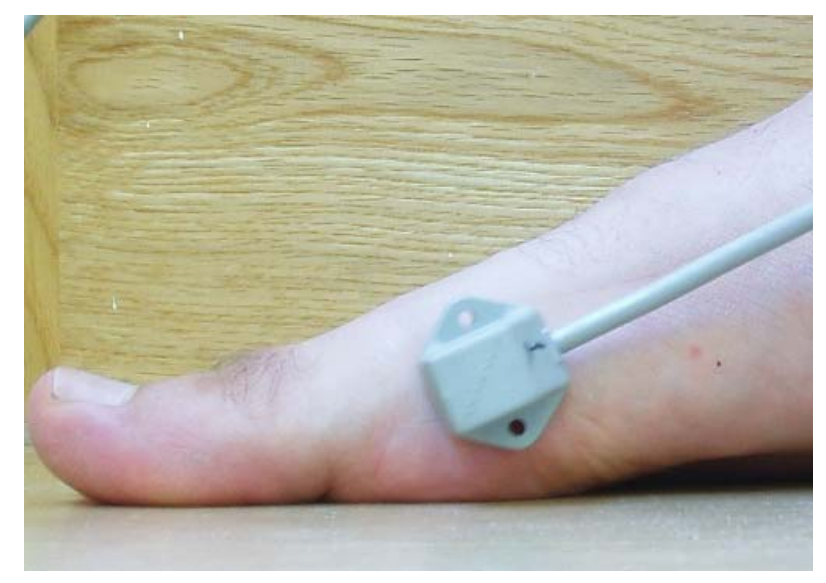

Figure 2. Sensor one. Location placed on the medial head of first metatarsal bone.

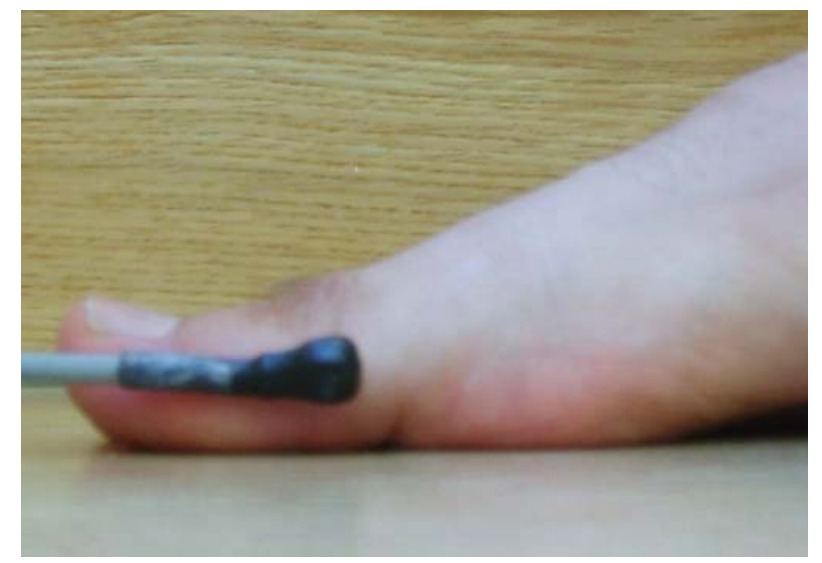

Figure 3. Sensor two. Location placed on the border of the proximal phalanx of the hallux. 


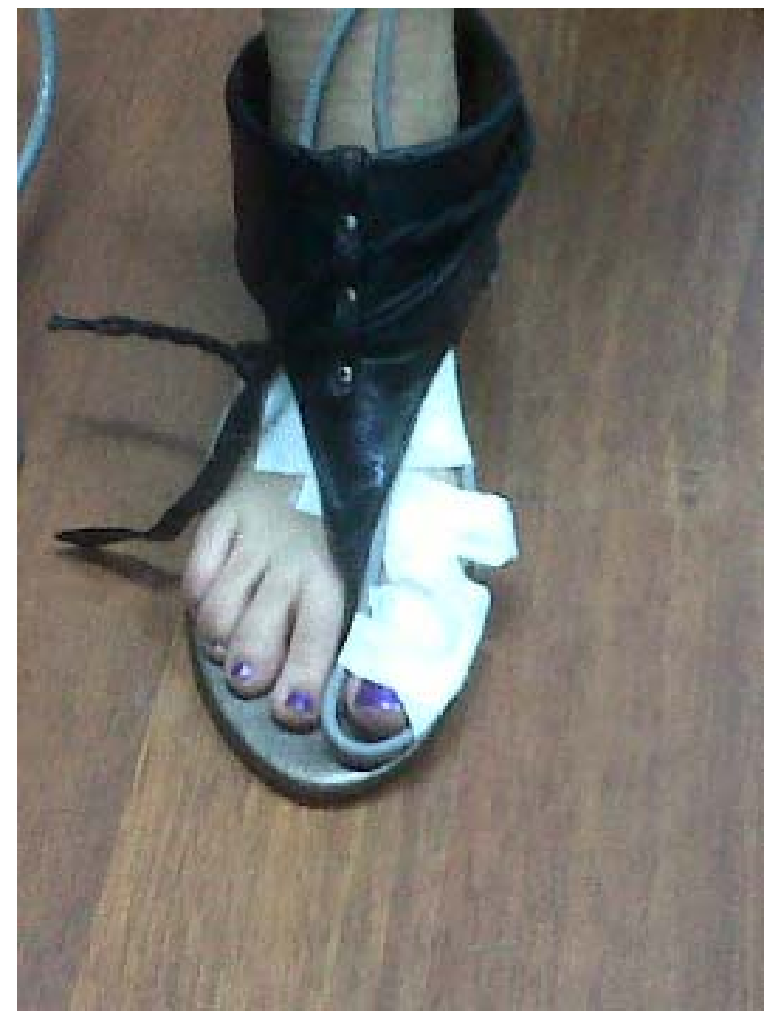

Figure 4. Fastening of the sensors. White bandages are auto-adhesive fixatives.

\subsection{Procedure}

Each of 80 participants began static or dynamic study randomly after choosing a sealed envelope that assigned them to one group or another; there were 40 total in each group. They then selected four sealed envelopes with each of the four conditions of the study (unshod, $3 \mathrm{~cm}, 6 \mathrm{~cm}$, and $9 \mathrm{~cm}$ ). These dictated the order of use at random. The 40 participants of the dynamic group did the static test in randomly order with four sealed envelopes and vice versa with the 40 participants in the static test (Figure 5).

Before beginning either angular measurements, position "zero" was achieved to calibrate the assessment and determine a reference position from which to begin the angular record. We asked the participants to remain in a relaxed standing position near the generated field for a few seconds until the software determined the Reference [35].

Participants used a pair of sandals with three different prefabricated high heels (Figure 6). The sandals (Figure 7) provided a strap in the first digital web space, and the rearfoot had a semi-rigid bowl that permitted the heels to be held into the shoes. The participants used the sandals and tested the sensations with the cables before starting the study; they then walked along the walkway at a self-selected speed. When they were comfortable, they began the static or dynamic measurements depending on the order chosen by the random chance.

For the dynamic condition study, participants initiated gait for $1 \mathrm{~m}$ at a self-selected speed before entering the $1.5 \mathrm{~m}$ calibrated capture volume. They then continued walking for a further $1.5 \mathrm{~m}$ (two steps); this capture was repeated for 5 trials with each of 3 different types of prefabricated heel heights $(3 \mathrm{~cm}, 6 \mathrm{~cm}$ and $9 \mathrm{~cm}$ ) and unshod condition. Care was taken when inserting and removing the prefabricated heel height so that the sensor devices was not disturbed or displaced. For the static condition study, subjects were asked to stand on their tiptoe (to reach the maximal range of motion of the joint) for $2 \mathrm{~s}$. There were five trials with each of three different types of prefabricated heel heights $(3 \mathrm{~cm}, 6 \mathrm{~cm}$ and $9 \mathrm{~cm}$ ) and unshod condition. The mean of the five trials was used in the posterior analysis for each static and dynamic test. 


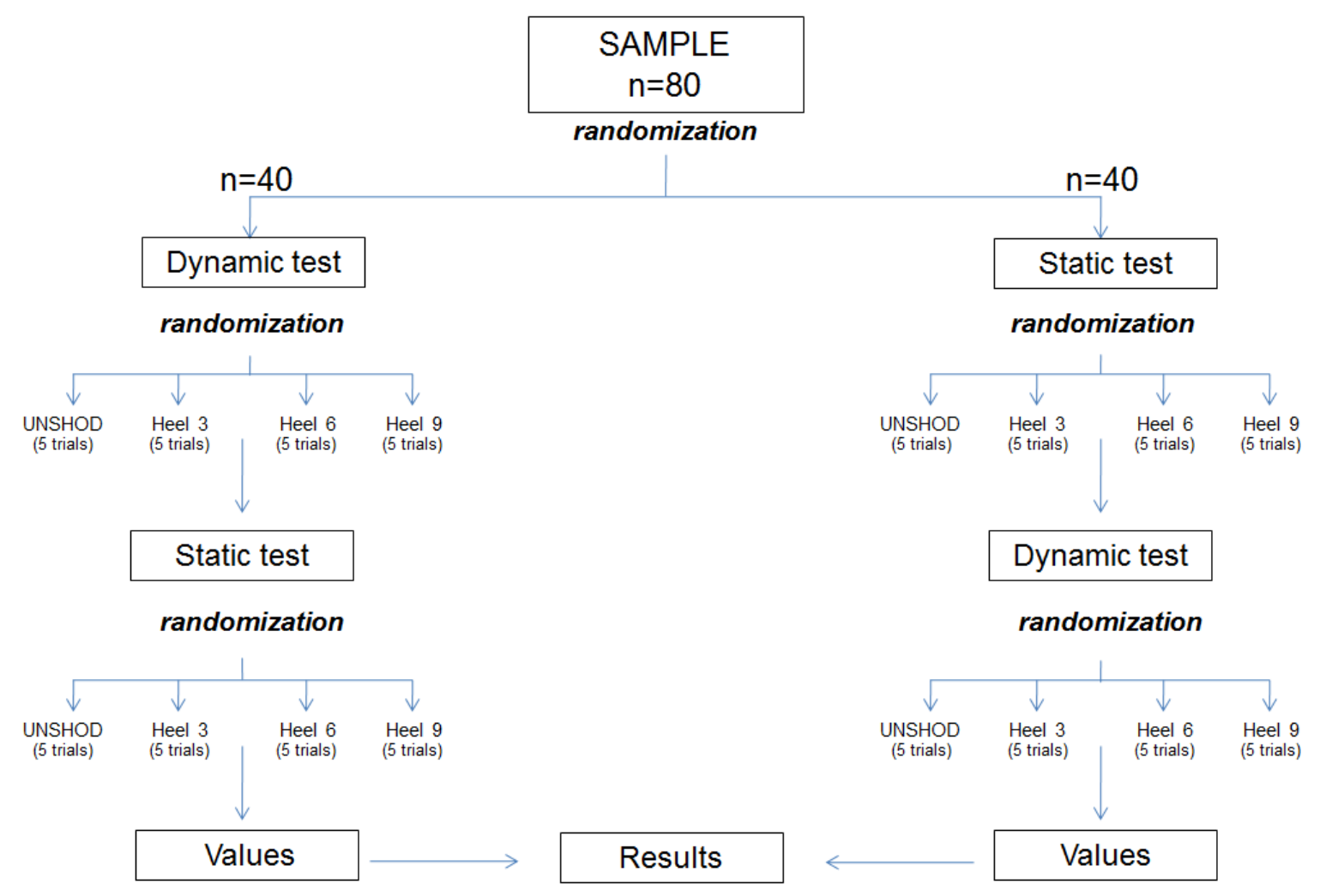

Figure 5. Randomized flow chart.

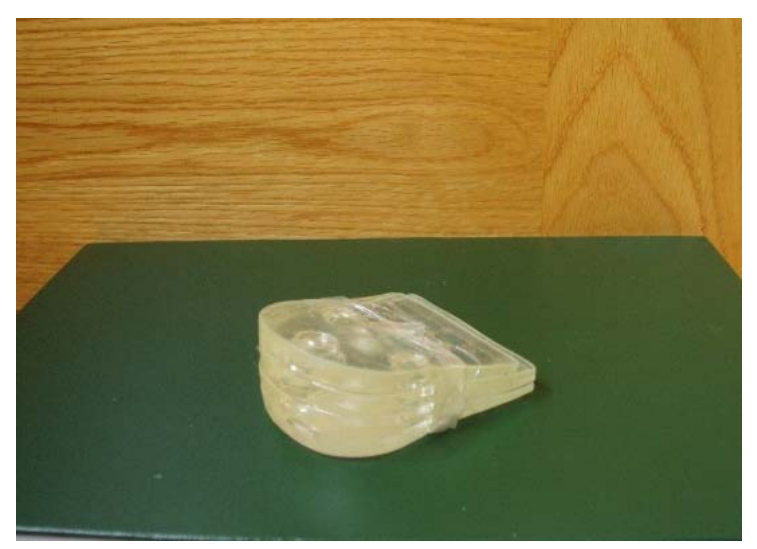

Figure 6. Prefabricated high-heels. The heels were made on hard silicone. The additional height was added over the top.

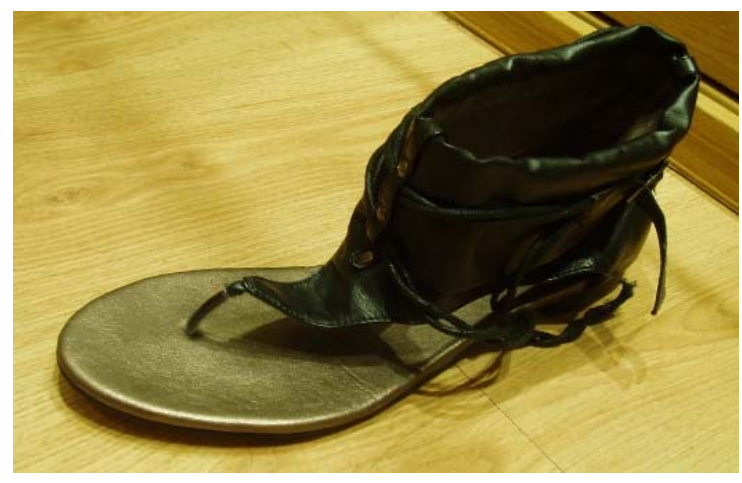

Figure 7. Sandal model used in the study. A free toe-tip-box is present to allow movement of proximal phalanx of the hallux and first metatarsal bone. 
Later, a "cardan system" with three possibilities of rotation (X-Y-Z) in two ways was used to draw angular and linear information from both PPH and IMTT bones movements: mediolateral axis $(\mathrm{X})$, defined dorsiflexion (DF), and plantar flexion (PF) movements on the sagittal plane as well as frontal plane inversion $(\mathrm{VR})$ and eversion $(\mathrm{VL})$ movements through an antero-posterior axis $(\mathrm{Y})$. Movement around a craneocaudal axis (Z) was considered to be abduction (ABD) and adduction (ADD) movements on a transverse plane. The anatomical landmarks and coordinate systems were previously detailed $[35,44,45]$. All bone movements were recorded after considering the laboratory coordinate system. All assessments used the same heel height in contralateral extremity under the foot to maintain the body balance during these trials [46]. Independent assessment of the bone movements showed that the results were only produced by high heel effects and not by bone interference.

\subsection{Statistical Analysis}

We recorded the within-day trial-to-trial intraclass correlation coefficients (ICC) and standard errors of measurement (SEM) [47] values for the participants wearing the different heel height (T3, T6, T9) vs. unshot in each plane of motion for PPH bone and IMTT bone in static and dynamic conditions. Landis and Koch [47] proposed that coefficients below 0.20 indicate slight agreement, and coefficients from 0.20 to 0.40 indicate fair reliability. Coefficients from 0.41 to 0.60 indicate moderate reliability, coefficients from 0.61 to 0.80 indicate substantial reliability, and coefficients from 0.81 to 1.00 indicate almost perfect reliability. We considered coefficients of 0.90 or larger to reflect a sufficient magnitude of reliability because they increase the likelihood that a measure is also reasonably valid. To check the concordance correlation between high heels' variables, Concordance Correlation Coefficient (CCC) [48] was done, where statistically significant $p$-values $(<0.05)$ would mean a perfect correlation [48]. SEM was utilized to determine the minimal detectable change (MDC) for all evaluations. This was also considered the Reliable Change Index (RCI). We utilized the RCI as a statistical method to determine the clinical significance according to Jacobson and Truax [49].

An initial Kolmogorov-Smirnov test showed that the data was not normally distributed $(p<0.05)$. The $p$-values for multiple comparisons were corrected with a non-parametric paired Friedman test to prove that all high-heels variables were different. Bivariate correlations with a Wilcoxon test were carried out to determine whether there were significant differences between "unshod" vs. "with high heels of T3, T6 and T9" in static and dynamic conditions in FPH and IMTT bones; an alpha level of 0.01 was established for all tests of significance. All data were studied to establish the effect of heel height in 3 axes of movement with the medial line of the body taken as reference movement. In addition, Spearman's Rho $(\rho)$ rank correlation coefficient both in static and dynamic conditions was done to check the possible relation between the height of the heels and the different bones' deviations. The results showed descriptive summaries as the mean \pm SD. Analyses of total 96 variables led to $p$-values $<0.05$ (within a $95 \%$ confidence interval) that considered statistically significant. We conducted data analysis with SPSS software version 19.0 (SPSS Science, Chicago, IL, USA).

\section{Results}

None of the data were normally distributed $(p<0.05)$. The means that the 96 measured variables were different when compared between each other $(p<0.001)$. Participants in this cross-sectional study were recruited from an orthopedic clinic in Madrid (Spain) over a 2-year period (May 2015 to May 2017). Of the 163 subjects who initially volunteered to participate in the study, 68 subjects did not meet the inclusion criteria. An additional 15 subjects did not present for testing. The remaining 80 subjects participated in the study (Figure 8). The participants were only females; sociodemographic data are shown in Table 1.

All Tables are shown with corresponding values of IMTT and PPH to the static test as well as independent of the dynamic values. 


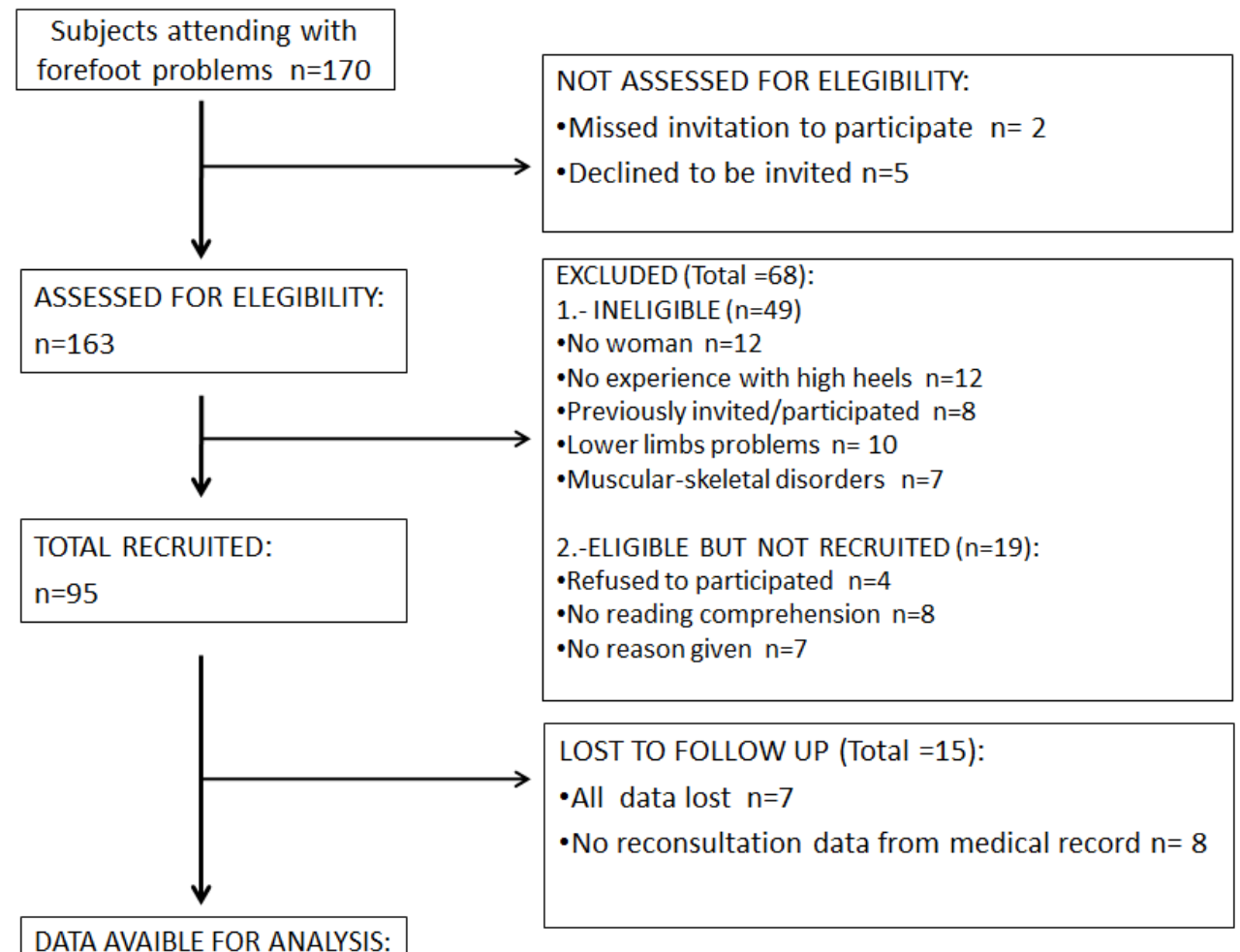
$n=80$

Figure 8. Participant flow chart.

Table 1. Sociodemographic characteristics of the participants.

\begin{tabular}{cc}
\hline \multirow{2}{*}{ Variable } & Female $\boldsymbol{n}=\mathbf{8 0}$ \\
\cline { 2 - 2 } & Mean \pm SD (95\% CI) \\
\hline Age (years) & $25.1 \pm 4.67(26.11-24.08)$ \\
Height $(\mathrm{cm})$ & $164.03 \pm 5.44(165.2-162.85)$ \\
Weight $(\mathrm{kg})$ & $57.53 \pm 6.304(58.90-56.15)$ \\
Foot Size $(\mathrm{EC})$ & $38.13 \pm 1.184(38.37-37.88)$ \\
BMI $\left(\mathrm{kg} / \mathrm{m}^{2}\right)$ & $20.2 \pm 1.74(20.58-19.82)$ \\
\hline
\end{tabular}

Abbreviations: $\quad \mathrm{EC}=$ European countries; $\mathrm{BMI}=$ body mass index; $\mathrm{SD}=$ Standard Deviation; $\mathrm{CI}=$ Confidence Interval.

The reliability of the static variables in unshod and heels of $3 \mathrm{~cm}, 6 \mathrm{~cm}$ and $9 \mathrm{~cm}$ high are summarized in Table 2. Each condition showed overall excellent reliability [50] with ICCs ranging from 0.902 to 0.997 indicating high reliability. In addition, in general terms, CCC showed a strong statistically significant correlation $(p<0.001)$ between unshod and either high heel in all movements of PPH and MTT except those related to a sagittal plane. The reliability of dynamic variables in unshod subjects for heel heights of $3 \mathrm{~cm}, 6 \mathrm{~cm}$ and $9 \mathrm{~cm}$ is summarized in Table 3. Each condition showed an overall excellent reliability [50] with ICCs ranging from 0.898 to 0.999 indicating high reliability. The CCC showed a strong correlation $(p<0.001)$ in all movements of PPH and MTT in dynamic tests, as well. The inter-rater MDC $95 \%$ values to static (Table 2) conditions ranged from $14.915^{\circ}$ to $0.74^{\circ}$ for sensor 1 (IMTT) and from $7.90^{\circ}$ to $1.63^{\circ}$ for sensor 2 (PPH); the inter-rater MDC $95 \%$ values to dynamic (Table 3) conditions ranged from $26.78^{\circ}$ to $1.12^{\circ}$ for sensor 1 (MTT) and from $27.16^{\circ}$ to $2.16^{\circ}$ for sensor $2(\mathrm{PPH})$.

The SEM values for $6 \mathrm{~cm}$ high heel in static abduction of PPH (Table 2) was $0.591^{\circ}$ and $0.782^{\circ}$ for dynamic abduction condition (Table 3); in addition, the SEM was $2.02^{\circ}$ for valgus movement of PPH in dynamic condition with high heels of $6 \mathrm{~cm}$ and $2.33^{\circ}$ in static condition. 
Table 2. Reliability ICC and CCC of static variables in unshod versus heels that were $3 \mathrm{~cm}, 6 \mathrm{~cm}$, or $9 \mathrm{~cm}$ high.

\begin{tabular}{|c|c|c|c|c|c|c|c|c|c|c|c|c|c|}
\hline \multirow{3}{*}{ Variable } & \multirow{3}{*}{$\begin{array}{c}\text { Unshod } \\
\text { ICC } \\
(95 \% C I)\end{array}$} & \multirow{3}{*}{$\begin{array}{c}\text { Heel } 3 \mathrm{~cm} \\
\text { ICC } \\
(95 \% \mathrm{CI})\end{array}$} & \multicolumn{3}{|c|}{ Unshod-Heel $3 \mathrm{~cm}$} & \multirow{3}{*}{$\begin{array}{c}\text { Heel } 6 \mathrm{~cm} \\
\text { ICC } \\
(95 \% \mathrm{CI})\end{array}$} & \multicolumn{3}{|c|}{ Unshod-Heel $6 \mathrm{~cm}$} & \multirow{3}{*}{$\begin{array}{c}\text { Heel } 9 \mathrm{~cm} \\
\text { ICC } \\
(95 \% \mathrm{CI})\end{array}$} & \multicolumn{3}{|c|}{ Unshod-Heel $9 \mathrm{~cm}$} \\
\hline & & & \multirow{2}{*}{ CCC $(95 \% \mathrm{CI})$} & \multirow{2}{*}{ SEM } & \multirow{2}{*}{$\begin{array}{c}\text { MDC } \\
95 \%\end{array}$} & & \multirow{2}{*}{ CCC $(95 \% \mathrm{CI})$} & \multirow{2}{*}{ SEM } & \multirow{2}{*}{$\begin{array}{c}\text { MDC } \\
95 \%\end{array}$} & & \multirow{2}{*}{ CCC $(95 \% \mathrm{CI})$} & \multirow{2}{*}{ SEM } & \multirow{2}{*}{$\begin{array}{r}\text { MDC } \\
95 \%\end{array}$} \\
\hline & & & & & & & & & & & & & \\
\hline MTT-AD & $\begin{array}{c}0.986 \\
(0.981-0.991)\end{array}$ & $\begin{array}{c}0.968 \\
(0.983-0.991)\end{array}$ & $\begin{array}{c}0.612 \\
(0.493-0.7)^{* *}\end{array}$ & 1.14 & 3.161 & $\begin{array}{c}0.991 \\
(0.988-0.994)\end{array}$ & $\begin{array}{c}0.477 \\
(0.341-0.594) * *\end{array}$ & 1.275 & 3.533 & $\begin{array}{c}0.984 \\
(0.978-0.0989) \\
\end{array}$ & $\begin{array}{c}0.184 \\
(0.106-0.26)^{* *}\end{array}$ & 1.455 & 4.034 \\
\hline MTT-AB & $\begin{array}{c}0.904 \\
(0.866-0.933)\end{array}$ & $\begin{array}{c}0.936 \\
(0.91-0.956)\end{array}$ & $\begin{array}{c}0.15 \\
(0.04-0.33)^{* *}\end{array}$ & 0.64 & 1.774 & $\begin{array}{c}0.985 \\
(0.965-0.862)\end{array}$ & $\begin{array}{c}0.258 \\
(0.042-0.45)\end{array}$ & 0.598 & 1.658 & $\begin{array}{c}0.961 \\
(0.945-0.973)\end{array}$ & $\begin{array}{c}0.136 \\
(0.012-0.277)^{* *}\end{array}$ & 0.711 & 1.97 \\
\hline PPH-AD & $\begin{array}{c}0.981 \\
(0.964-0.992) \\
\end{array}$ & $\begin{array}{c}0.95 \\
(0.93-0.965)\end{array}$ & $\begin{array}{c}0.096 \\
(0.039-0.153)^{* *}\end{array}$ & 1.357 & 3.762 & $\begin{array}{c}0.969 \\
(0.956-0.978) \\
\end{array}$ & $\begin{array}{c}0.054 \\
(0.11-0.13)^{* *} \\
\end{array}$ & 1.65 & 4.574 & $\begin{array}{c}0.944 \\
(0.922-0.961) \\
\end{array}$ & $\begin{array}{c}0.147 \\
(0.081-0.212) \\
\end{array}$ & 1.298 & 3.598 \\
\hline PPH-ABD & $\begin{array}{c}0.94 \\
(0.916-0.958) \\
\end{array}$ & $\begin{array}{c}0.908 \\
(0.872-0.937) \\
\end{array}$ & $\begin{array}{c}0.006 \\
(0.01-0.019) * *\end{array}$ & 0.828 & 2.296 & $\begin{array}{c}0.926 \\
(0.897-0.949) \\
\end{array}$ & $\begin{array}{c}0.239 \\
(0.040-0.41)\end{array}$ & 0.591 & 1.639 & $\begin{array}{c}0.969 \\
(0.957-0.979)\end{array}$ & $\begin{array}{c}0.168 \\
(0.044-0.365)^{* *}\end{array}$ & 0.63 & 1.747 \\
\hline MTT-PF & $\begin{array}{c}0.997 \\
(0.995-0.998)\end{array}$ & $\begin{array}{c}0.994 \\
(0.992-0.996)\end{array}$ & $\begin{array}{c}0.792 \\
(0.727-0.842)\end{array}$ & 2.493 & 6.911 & $\begin{array}{c}0.991 \\
(0.988-0.994)\end{array}$ & $\begin{array}{c}0.162 \\
(0.033-0.286)\end{array}$ & 5.633 & 15.615 & $\begin{array}{c}0.99 \\
(0.986-0.993)\end{array}$ & $\begin{array}{c}0.19 \\
(0.104-0.272) \text { * }\end{array}$ & 5.381 & 14.915 \\
\hline MTT-DF & $\begin{array}{c}0.902 \\
(0.864-0.932)\end{array}$ & $\begin{array}{c}0.928 \\
(0.90-0.95)\end{array}$ & $\begin{array}{c}0.414 \\
(0.219-0.577) \\
\end{array}$ & 0.304 & 0.844 & $\begin{array}{c}0.918 \\
(0.886-0.944)\end{array}$ & $\begin{array}{c}0.487 \\
(0.30-0.63)\end{array}$ & 0.27 & 0.749 & $\begin{array}{c}0.968 \\
(0.955-0.978)\end{array}$ & $\begin{array}{c}0.107 \\
(0.008-0.218) \text { * }\end{array}$ & 0.579 & 1.604 \\
\hline PPH-PF & $\begin{array}{c}0.983 \\
(0.977-0.989)\end{array}$ & $\begin{array}{c}0.982 \\
(0.974-0.987)\end{array}$ & $\begin{array}{c}0.034 \\
(0.16-0.23)\end{array}$ & 1.825 & 5.059 & $\begin{array}{c}0.988 \\
(0.984-0.992)\end{array}$ & $\begin{array}{c}0.091 \\
(0.10-0.279)\end{array}$ & 1.67 & 4.63 & $\begin{array}{c}0.979 \\
(0.971-0.986)\end{array}$ & $\begin{array}{c}0.19 \\
(0.013-0.356)^{* *}\end{array}$ & 1.574 & 4.363 \\
\hline PPH-DF & $\begin{array}{c}0.956 \\
(0.939-0.970)\end{array}$ & $\begin{array}{c}0.978 \\
(0.969-0.984)\end{array}$ & $\begin{array}{c}0.381 \\
(0.182-0.549)\end{array}$ & 1.213 & 3.361 & $\begin{array}{c}0.99 \\
(0.986-0.993)\end{array}$ & $\begin{array}{c}0.26 \\
(0.066-0.435)\end{array}$ & 1.628 & 4.512 & $\begin{array}{c}0.982 \\
(0.975-0.988)\end{array}$ & $\begin{array}{c}0.153 \\
(0.037-0.67)^{* *}\end{array}$ & 2.274 & 6.303 \\
\hline MTT-VL & $\begin{array}{c}0.975 \\
(0.965-0.983) \\
\end{array}$ & $\begin{array}{c}0.994 \\
(0.992-0.996) \\
\end{array}$ & $\begin{array}{c}0.043 \\
(0.004-0.091)^{* *} \\
\end{array}$ & 2.471 & 6.848 & $\begin{array}{c}0.961 \\
(0.946-0.983) \\
\end{array}$ & $\begin{array}{c}0.158 \\
(0.069-0.244)\end{array}$ ** & 1.202 & 3.333 & $\begin{array}{c}0.973 \\
(0.962-0.981)\end{array}$ & $\begin{array}{c}0.082 \\
(0.033-0.13)^{* *}\end{array}$ & 1.277 & 3.539 \\
\hline MTT-VR & $\begin{array}{c}0.995 \\
(0.993-0.996)\end{array}$ & $\begin{array}{c}0.976 \\
(0.967-0.984)\end{array}$ & $\begin{array}{c}0.178 \\
(0.29-0.31) \text { * }\end{array}$ & 2.419 & 6.706 & $\begin{array}{c}0.99 \\
(0.986-0.993)\end{array}$ & $\begin{array}{c}0.64 \\
(0.513-0.739) * *\end{array}$ & 1.463 & 4.054 & $\begin{array}{c}0.971 \\
(0.960-0.98)\end{array}$ & $\begin{array}{c}0.43 \\
(0.33-0.52)^{* *}\end{array}$ & 1.463 & 4.747 \\
\hline PPH-VL & $\begin{array}{c}0.989 \\
(0.985-0.993) \\
\end{array}$ & $\begin{array}{c}0.993 \\
(0.99-0.995) \\
\end{array}$ & $\begin{array}{c}0.455 \\
(0.328-0.567) * *\end{array}$ & 2.023 & 5.608 & $\begin{array}{c}0.985 \\
(0.979-0.990) \\
\end{array}$ & $\begin{array}{c}0.162 \\
(0.035-0.347)\end{array}$ & 2.336 & 6.476 & $\begin{array}{c}0.981 \\
(0.974-0.987) \\
\end{array}$ & $\begin{array}{c}0.128 \\
(0.051-0.299) * *\end{array}$ & 2.143 & 5.94 \\
\hline PPH-VR & $\begin{array}{c}0.98 \\
(0.972-0.986)\end{array}$ & $\begin{array}{c}0.978 \\
(0.969-0.985)\end{array}$ & $\begin{array}{c}0.388 \\
(0.198-0.55)\end{array}$ & 1.395 & 3.866 & $\begin{array}{c}0.975 \\
(0.966-0.983)\end{array}$ & $\begin{array}{c}0.152 \\
(0.058-0.35)^{* *}\end{array}$ & 1.921 & 5.326 & $\begin{array}{c}0.989 \\
(0.984-0.992)\end{array}$ & $\begin{array}{c}0.13 \\
(0.047-0.18)^{* *}\end{array}$ & 2.851 & 7.902 \\
\hline
\end{tabular}

Abbreviations: ICC = Intraclass Correlation Coefficient; $\mathrm{CI}=$ Confidence Interval; $\mathrm{CCC}=$ Concordance Correlation Coefficient; SEM = standard error of measurement; MDC = minimal detectable change; MTT ADD = first metatarsal bone in Adduction, I MTT toward to medial body line; MTT ABD = first metatarsal bone in Abduction, I MTT away to medial body line; MTT PF = first metatarsal bone in plantarflexion, I MTT toward to the floor; MTT DF = first metatarsal bone in dorsiflexion, I MTT away to the floor; MTT VL = first metatarsal bone spins to inside, toward medial body line; MTT VR = first metatarsal bone spin to outside, away to medial body line; PPH AD = proximal phalanx of hallux in adduction: PPH toward to medial body line; PPH ABD = proximal phalanx of hallux in abduction: PPH away to medial body line; PPH PF = proximal phalanx of hallux in plantarflexion: PPH toward to the floor; PPH DF = proximal phalanx of hallux in dorsiflexion: $\mathrm{PPH}$ away to the floor; PPH VL = proximal phalanx of hallux spins to inside: PPH toward medial body line; PPH VR = proximal phalanx of hallux spin to outside: PPH away to medial body line; UNSHOD = barefoot participants without any height in shoe heel; \pm SD = standard deviation. Movements and axes refer to the medial line of the body; all values are expressed in degrees; ${ }^{*} p$-value $<0.05 ;{ }^{* *} p$-value $<0.001$ 
Table 3. Reliability ICC and CCC of dynamic variables in unshod versus heels that were $3 \mathrm{~cm}, 6 \mathrm{~cm}$, or $9 \mathrm{~cm}$ high.

\begin{tabular}{|c|c|c|c|c|c|c|c|c|c|c|c|c|c|}
\hline \multirow{3}{*}{ Variable } & \multirow{3}{*}{$\begin{array}{c}\text { Unshod } \\
\text { ICC } \\
(95 \% \mathrm{CI})\end{array}$} & \multirow{3}{*}{$\begin{array}{c}\text { Heel } 3 \mathrm{~cm} \\
\text { ICC } \\
(95 \% \mathrm{CI})\end{array}$} & \multicolumn{3}{|c|}{ Unshod-Heel $3 \mathrm{~cm}$} & \multirow{3}{*}{$\begin{array}{c}\text { Heel } 6 \mathrm{~cm} \\
\text { ICC } \\
(95 \% \mathrm{CI})\end{array}$} & \multicolumn{3}{|c|}{ Unshod-Heel $6 \mathrm{~cm}$} & \multirow{3}{*}{$\begin{array}{c}\text { Heel } 9 \mathrm{~cm} \\
\text { ICC } \\
(95 \% \mathrm{CI})\end{array}$} & \multicolumn{3}{|c|}{ Unshod-Heel $9 \mathrm{~cm}$} \\
\hline & & & \multirow{2}{*}{ CCC $(95 \% \mathrm{CI})$} & \multirow{2}{*}{ SEM } & \multirow{2}{*}{$\begin{array}{c}\text { MDC } \\
95 \%\end{array}$} & & \multirow{2}{*}{ CCC $(95 \% \mathrm{CI})$} & \multirow{2}{*}{ SEM } & \multirow{2}{*}{$\begin{array}{c}\text { MDC } \\
95 \%\end{array}$} & & \multirow{2}{*}{ CCC $(95 \% \mathrm{CI})$} & \multirow{2}{*}{ SEM } & \multirow{2}{*}{$\begin{array}{c}\text { MDC } \\
95 \%\end{array}$} \\
\hline & & & & & & & & & & & & & \\
\hline MTT-AD & $\begin{array}{c}0.988 \\
(0.983-0.992)\end{array}$ & $\begin{array}{c}0.986 \\
(0.981-0.990)\end{array}$ & $\begin{array}{c}0.191 \\
(0.123-0.25) * *\end{array}$ & 1.953 & 5.414 & $\begin{array}{c}0.968 \\
(0.955-0.978)\end{array}$ & $\begin{array}{c}0.25 \\
(0.171-0.327) * *\end{array}$ & 1.785 & 4.949 & $\begin{array}{c}0.986 \\
(0.981-0.99)\end{array}$ & $\begin{array}{c}0.232 \\
(0.15-0.30)\end{array}$ & 1.813 & 5.026 \\
\hline MTT-AB & $\begin{array}{c}0.993 \\
(0.991-0.995)\end{array}$ & $\begin{array}{c}0.993 \\
(0.990-0.995)\end{array}$ & $\begin{array}{c}0.041 \\
(0.48-0.131)\end{array}$ & 3.529 & 9.782 & $\begin{array}{c}0.997 \\
(0.995-0.998)\end{array}$ & $\begin{array}{c}0.151 \\
(0.055-0.245) *\end{array}$ & 3.499 & 9.699 & $\begin{array}{c}0.992 \\
(0.988-0.944)\end{array}$ & $\begin{array}{c}0.28 \\
(0.18-0.46)\end{array}$ & 2.715 & 7.526 \\
\hline PPH-AD & $\begin{array}{c}0.938 \\
(0.913-0.957)\end{array}$ & $\begin{array}{c}0.958 \\
(0.941-0.971)\end{array}$ & $\begin{array}{c}0.049 \\
(0.11-0.17) * *\end{array}$ & 1.051 & 2.914 & $\begin{array}{c}0.965 \\
(0.951-0.976)\end{array}$ & $\begin{array}{c}0.026 \\
(0.10-0.157)^{* *}\end{array}$ & 1.334 & 3.698 & $\begin{array}{c}0.977 \\
(0.969-0.984)\end{array}$ & $\begin{array}{c}0.197 \\
(0.06-0.321) *\end{array}$ & 1.117 & 3.096 \\
\hline PPH-ABD & $\begin{array}{c}0.976 \\
(0.966-0.983) \\
\end{array}$ & $\begin{array}{c}0.983 \\
(0.976-0.988) \\
\end{array}$ & $\begin{array}{c}0.04 \\
(0.03-0.11)^{* *} \\
\end{array}$ & 1.852 & 5.132 & $\begin{array}{c}0.981 \\
(0.973-0.987) \\
\end{array}$ & $\begin{array}{c}0.51 \\
(0.33-0.65) * * \\
\end{array}$ & 0.782 & 2.169 & $\begin{array}{c}0.994 \\
(0.991-0.996) \\
\end{array}$ & $\begin{array}{c}0.295 \\
(0.17-0.41)^{* *} \\
\end{array}$ & 1.793 & 4.971 \\
\hline MTT-PF & $\begin{array}{c}0.998 \\
(0.998-0.999)\end{array}$ & $\begin{array}{c}0.997 \\
(0.996-0.998)\end{array}$ & $\begin{array}{c}0.834 \\
(0.77-0.88)\end{array}$ & 4.421 & 12.255 & $\begin{array}{c}0.998 \\
(0.997-0.999)\end{array}$ & $\begin{array}{c}0.799 \\
(0.714-0.861)\end{array}$ & 5.051 & 14.001 & $\begin{array}{c}0.992 \\
(0.988-0.994)\end{array}$ & $\begin{array}{c}0.268 \\
(0.14-0.38)\end{array}$ & 9.662 & 26.782 \\
\hline MTT-DF & $\begin{array}{c}0.935 \\
(0.910-0.955) \\
\end{array}$ & $\begin{array}{c}0.898 \\
(0.857-0.929)\end{array}$ & $\begin{array}{c}0.356 \\
(0.186-0.521) \\
\end{array}$ & 0.404 & 1.121 & $\begin{array}{c}0.933 \\
(0.907-0.954)\end{array}$ & $\begin{array}{c}0.198 \\
(0.015-0.368) \\
\end{array}$ & 0.416 & 1.153 & $\begin{array}{c}0.953 \\
(0.935-0.968)\end{array}$ & $\begin{array}{c}0.453 \\
(0.262-0.61)\end{array}$ & 0.417 & 1.155 \\
\hline PPH-PF & $\begin{array}{c}0.999 \\
(0.999-1.00)\end{array}$ & $\begin{array}{c}0.999 \\
(0.998-0.999)\end{array}$ & $\begin{array}{c}0.723 \\
(0.608-0.808)\end{array}$ & 6.755 & 18.723 & $\begin{array}{c}0.998 \\
(0.998-0.999)\end{array}$ & $\begin{array}{c}0.857 \\
(0.787-0.9)\end{array}$ & 4.929 & 13.662 & $\begin{array}{c}0.996 \\
(0.995-0.997)\end{array}$ & $\begin{array}{c}0.425 \\
(0.298-0.538)\end{array}$ & 9.801 & 27.166 \\
\hline PPH-DF & $\begin{array}{c}0.981 \\
(0.974-0.987)\end{array}$ & $\begin{array}{c}0.917 \\
(0.844-0.943)\end{array}$ & $\begin{array}{c}0.175 \\
(0.002-0.185)\end{array}$ & 1.181 & 3.272 & $\begin{array}{c}0.928 \\
(0.9-0.950)\end{array}$ & $\begin{array}{c}0.198 \\
(0.015-0.36)\end{array}$ & 1.17 & 3.243 & $\begin{array}{c}0.958 \\
(0.941-0.971)\end{array}$ & $\begin{array}{c}0.231 \\
(0.038-0.40) *\end{array}$ & 1.195 & 3.313 \\
\hline MTT-VL & $\begin{array}{c}0.985 \\
(0.979-0.990) \\
\end{array}$ & $\begin{array}{c}0.99 \\
(0.986-0.993) \\
\end{array}$ & $\begin{array}{c}0.137 \\
(0.020-0.250) \text { ** }\end{array}$ & 2.326 & 6.446 & $\begin{array}{c}0.994 \\
(0.992-0.996) \\
\end{array}$ & $\begin{array}{c}0.075 \\
(0.05-0.2) * *\end{array}$ & 2.997 & 8.306 & $\begin{array}{c}0.993 \\
(0.991-0.995)\end{array}$ & $\begin{array}{c}0.12 \\
(0.017-0.21)^{* *} \\
\end{array}$ & 2.565 & 7.109 \\
\hline MTT-VR & $\begin{array}{c}0.985 \\
(0.979-0.989) \\
\end{array}$ & $\begin{array}{c}0.982 \\
(0.975-0.988)\end{array}$ & $\begin{array}{c}0.26 \\
(0.183-0.334)^{* *}\end{array}$ & 2.314 & 6.413 & $\begin{array}{c}0.947 \\
(0.927-0.964)\end{array}$ & $\begin{array}{c}0.15 \\
(0.084-0.214) \\
\end{array}$ & 2.476 & 6.864 & $\begin{array}{c}0.928 \\
(0.9-0.951)\end{array}$ & $\begin{array}{c}0.042 \\
(0.04-0.05)\end{array}$ & 2.703 & 7.491 \\
\hline PPH-VL & $\begin{array}{c}0.964 \\
(0.950-0.975) \\
\end{array}$ & $\begin{array}{c}0.99 \\
(0.986-0.993) \\
\end{array}$ & $\begin{array}{c}0.392 \\
(0.293-0.484)^{* *}\end{array}$ & 1.928 & 5.343 & $\begin{array}{c}0.986 \\
(0.981-0.99) \\
\end{array}$ & $\begin{array}{c}0.225 \\
(0.131-0.315)^{* *}\end{array}$ & 2.022 & 5.605 & $\begin{array}{c}0.969 \\
(0.956-0.978) \\
\end{array}$ & $\begin{array}{c}0.12 \\
(0.011-0.246)\end{array}$ & 2.439 & 6.76 \\
\hline PPH-VR & $\begin{array}{c}0.992 \\
(0.989-0.995)\end{array}$ & $\begin{array}{c}0.987 \\
(0.981-0.991)\end{array}$ & $\begin{array}{c}0.53 \\
(0.364-0.664)^{* *}\end{array}$ & 1.535 & 4.256 & $\begin{array}{c}0.951 \\
(0.931-0.966)\end{array}$ & $\begin{array}{c}0.384 \\
(0.265-0.491)^{* *}\end{array}$ & 1.577 & 4.37 & $\begin{array}{c}0.981 \\
(0.9-0.951)\end{array}$ & $\begin{array}{c}0.472 \\
(0.291-0.621)\end{array}$ & 1.656 & 4.591 \\
\hline
\end{tabular}

Abbreviations: ICC = Intraclass Correlation Coefficient; $\mathrm{CI}=$ Confidence Interval; $\mathrm{CCC}=$ Concordance Correlation Coefficient; SEM = standard error of measurement; MDC = minimal detectable change; MTT ADD = first metatarsal bone in Adduction, I MTT toward to medial body line; MTT ABD = first metatarsal bone in Abduction, I MTT away to medial body line; MTT PF = first metatarsal bone in plantarflexion, I MTT toward to the floor; MTT DF = first metatarsal bone in dorsiflexion, I MTT away to the floor; MTT VL = first metatarsal bone spins to inside, toward medial body line; MTT VR = first metatarsal bone spin to outside, away to medial body line; PPH AD = proximal phalanx of hallux in adduction: PPH toward to medial body line; PPH ABD = proximal phalanx of hallux in abduction: PPH away to medial body line; PPH PF = proximal phalanx of hallux in plantarflexion: PPH toward to the floor; PPH DF = proximal phalanx of hallux in dorsiflexion: PPH away to the floor; PPH VL = proximal phalanx of hallux spins to inside: PPH toward medial body line; PPH VR = proximal phalanx of hallux spin to outside: PPH away to medial body line; UNSHOD = barefoot participants without any height in shoe heel; \pm SD = standard deviation. Movements and axes refer to the medial line of the body; all values are expressed in degrees; ${ }^{*} p$-value $<0.05 ;{ }^{* *} p$-value $<0.001$ 
Static mobility grades of IMTT (sensor 1) and PPH (sensor 2) bones in unshod and $3 \mathrm{~cm}, 6 \mathrm{~cm}$ and $9 \mathrm{~cm}$ heel height are summarized in Table 4 . The dynamic mobility grades of both sensor 1 and sensor 2 in unshod and $3 \mathrm{~cm}, 6 \mathrm{~cm}$ and $9 \mathrm{~cm}$ heel heights in Table 5.

Under static conditions, the PPH abduction increases from $1.01 \pm 0.36^{\circ}$ to $1.31 \pm 0.46^{\circ}(p<0.05)$ after wearing shoes with a $3 \mathrm{~cm}$ heel. There was a statistically significant valgus of the I MTT with increasing heel heights from $1.77 \pm 0.20^{\circ}$ without high heels to $2.15 \pm 0.10^{\circ}$ with $3 \mathrm{~cm}(p<0.001)$ high heels. There was increased varus movement from $1.50 \pm 0.23$ to $3.87 \pm 0.20$ with $3 \mathrm{~cm}$ heels $(p<0.001)$.

In dynamic tests, wearing shoes with $6 \mathrm{~cm}$ high heels led to an increase in $\mathrm{PPH}$ valgus and abduction deviation from $3.15 \pm 0.10^{\circ}$ to $3.46 \pm 0.05^{\circ}(p<0.05)$ and from $1.35 \pm 0.28^{\circ}$ to $1.69 \pm 0.30^{\circ}$ $(p<0.001)$, respectively. In addition, PPH had abduction that increased to $1.91 \pm$ with $9 \mathrm{~cm}$ heels $(p<0.001)$. On the other hand, I MTT had a valgus decrease with $6 \mathrm{~cm}$ heels (from $3.94 \pm 0.28$ to $3.70 \pm 0.13(p<0.001))$ but without any concordance correlation.

Table 4. STATIC mobility grades of first metatarsal (sensor 1) and proximal phalanx of hallux (sensor 2) bones in unshod with heels of $3 \mathrm{~cm}, 6 \mathrm{~cm}$, and $9 \mathrm{~cm}$ high.

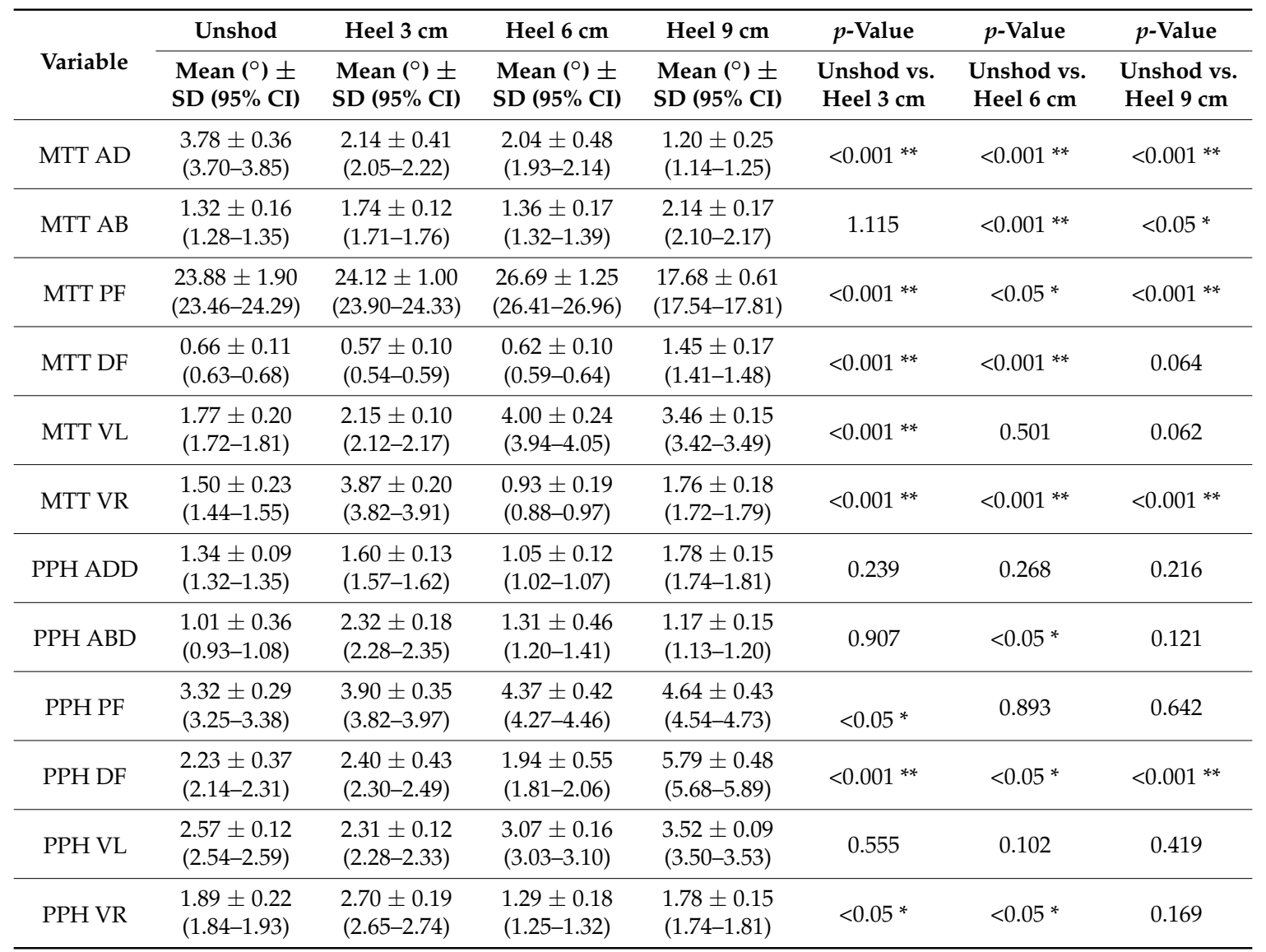

Abbreviations: MTT ADD = first metatarsal bone in Adduction: I MTT toward to medial body line; MTT ABD = first metatarsal bone in abduction: I MTT away to medial body line; MTT PF = first metatarsal bone in plantarflexion: I MTT toward to the floor; MTT DF = first metatarsal bone in dorsiflexion: I MTT away to the floor; MTT VL = first metatarsal bone spins to inside: I MTT toward medial body line; MTT VR = first metatarsal bone spin to outside: I MTT away to medial body line; UNSHOD = barefoot participants without any height in shoe heel; PPH ADD = proximal phalanx of hallux in adduction: PPH toward to medial body line; PPH ABD = proximal phalanx of hallux in Abduction: PPH away to medial body line; PPH PF = proximal phalanx of hallux in plantarflexion: PPH toward to the floor; PPH DF = proximal phalanx of hallux in dorsiflexion: PPH away to the floor; PPH VL = proximal phalanx of hallux spins to inside: PPH toward medial body line; PPH VR = proximal phalanx of hallux spin to outside: PPH away to medial body line; $\pm \mathrm{SD}$ = standard deviation; $p$-value $<0.05$ * (within a $95 \%$ confidence interval) was considered statistically significant; $p$-value $<0.001{ }^{* *}$ (within a $95 \%$ confidence interval) was considered statistically strong significant; Movements and axes refer to the medial line of the body; all values are expressed in degrees. 
Table 5. DYNAMIC mobility grades of first metatarsal (sensor 1) and proximal phalanx of hallux (sensor 2) bones in unshod as well as heels $3 \mathrm{~cm}, 6 \mathrm{~cm}$ and $9 \mathrm{~cm}$ high.

\begin{tabular}{|c|c|c|c|c|c|c|c|}
\hline \multirow[b]{2}{*}{ Variable } & Unshod & Heel $3 \mathrm{~cm}$ & Heel $6 \mathrm{~cm}$ & Heel $9 \mathrm{~cm}$ & $p$-Value & $p$-Value & $p$-Value \\
\hline & $\begin{array}{l}\text { Mean }\left({ }^{\circ}\right) \pm \\
\text { SD }(95 \% \mathrm{CI})\end{array}$ & $\begin{array}{l}\text { Mean }\left({ }^{\circ}\right) \pm \\
\text { SD }(95 \% \mathrm{CI})\end{array}$ & $\begin{array}{l}\text { Mean }\left({ }^{\circ}\right) \pm \\
\operatorname{SD}(95 \% \mathrm{CI})\end{array}$ & $\begin{array}{l}\text { Mean }\left({ }^{\circ}\right) \pm \\
\text { SD }(95 \% \text { CI })\end{array}$ & $\begin{array}{l}\text { Unshod vs. } \\
\text { Heel } 3 \mathrm{~cm}\end{array}$ & $\begin{array}{l}\text { Unshod vs. } \\
\text { Heel } 6 \mathrm{~cm}\end{array}$ & $\begin{array}{l}\text { Unshod vs. } \\
\text { Heel } 9 \mathrm{~cm}\end{array}$ \\
\hline MTT ADD & $\begin{array}{l}4.97 \pm 0.36 \\
(4.89-5.04)\end{array}$ & $\begin{array}{l}1.42 \pm 0.35 \\
(1.34-1.49)\end{array}$ & $\begin{array}{l}2.51 \pm 0.30 \\
(2.43-2.59)\end{array}$ & $\begin{array}{l}2.25 \pm 030 \\
(2.17-2.32)\end{array}$ & 0.226 & 0.088 & 0.852 \\
\hline MTT ABD & $\begin{array}{l}1.89 \pm 0.17 \\
(1.85-1.92)\end{array}$ & $\begin{array}{l}0.76 \pm 0.10 \\
(0.73-0.78)\end{array}$ & $\begin{array}{l}1.04 \pm 0.08 \\
(1.02-1.06)\end{array}$ & $\begin{array}{l}2.01 \pm 0.14 \\
(1.96-2.04)\end{array}$ & 0.211 & $<0.001 * *$ & $<0.05^{*}$ \\
\hline MTT PF & $\begin{array}{l}38.30 \pm 3.38 \\
(37.55-39.04)\end{array}$ & $\begin{array}{l}35.06 \pm 1.99 \\
(34.62-35.49)\end{array}$ & $\begin{array}{l}36.13 \pm 2.67 \\
(35.54-36.71)\end{array}$ & $\begin{array}{l}44.88 \pm 1.40 \\
(44.57-45.18)\end{array}$ & $<0.001 * *$ & $<0.001 * *$ & $<0.05^{*}$ \\
\hline MTT DF & $\begin{array}{l}0.97 \pm 0.15 \\
(0.93-1.00)\end{array}$ & $\begin{array}{l}0.83 \pm 0.10 \\
(0.80-0.85)\end{array}$ & $\begin{array}{l}0.86 \pm 0.13 \\
(0.83-0.89)\end{array}$ & $\begin{array}{l}0.81 \pm 0.14 \\
(0.76-0.86)\end{array}$ & $<0.001 * *$ & $<0.001 * *$ & $<0.001^{* *}$ \\
\hline MTT VL & $\begin{array}{l}3.94 \pm 0.28 \\
(3.87-4.00)\end{array}$ & $\begin{array}{l}2.88 \pm 0.10 \\
(2.85-2.90)\end{array}$ & $\begin{array}{l}3.70 \pm 0.13 \\
(3.67-3.73)\end{array}$ & $\begin{array}{l}3.60 \pm 0.08 \\
(3.58-3.61)\end{array}$ & 0.724 & $<0.001 * *$ & 0.526 \\
\hline MTT VR & $\begin{array}{l}3.92 \pm 0.19 \\
(3.85-3.94)\end{array}$ & $\begin{array}{l}1.11 \pm 0.21 \\
(1.06-1.16)\end{array}$ & $\begin{array}{l}1.08 \pm 0.15 \\
(1.04-1.11)\end{array}$ & $\begin{array}{l}2.27 \pm 0.26 \\
(2.21-2.32)\end{array}$ & 0.98 & 0.25 & 0.653 \\
\hline PPH ADD & $\begin{array}{l}2.39 \pm 0.13 \\
(2.36-2.41)\end{array}$ & $\begin{array}{l}0.87 \pm 0.15 \\
(0.83-0.90)\end{array}$ & $\begin{array}{l}1.31 \pm 0.14 \\
(1.26-1.33)\end{array}$ & $\begin{array}{l}1.12 \pm 0.19 \\
(1.07-1.16)\end{array}$ & $<0.001 * *$ & 0.088 & $<0.001 * *$ \\
\hline PPH ABD & $\begin{array}{l}1.35 \pm 0.28 \\
(1.28-1.41)\end{array}$ & $\begin{array}{l}4.56 \pm 0.50 \\
(4.45-4.66)\end{array}$ & $\begin{array}{l}1.69 \pm 0.30 \\
(1.66-1.71)\end{array}$ & $\begin{array}{l}1.91 \pm 0.24 \\
(1.85-1.96)\end{array}$ & 0.486 & $<0.001 * *$ & $<0.05^{*}$ \\
\hline PPH PF & $\begin{array}{l}8.89 \pm 0.48 \\
(8.78-8.99)\end{array}$ & $\begin{array}{l}4.66 \pm 0.10 \\
(4.63-4.69)\end{array}$ & $\begin{array}{l}8.87 \pm 0.48 \\
(8.76-8.97)\end{array}$ & $\begin{array}{l}2.25 \pm 0.21 \\
(2.20-2.29)\end{array}$ & 0.372 & $<0.001 * *$ & 0.605 \\
\hline PPH DF & $\begin{array}{l}1.97 \pm 0.39 \\
(1.88-2.05)\end{array}$ & $\begin{array}{l}1.67 \pm 0.20 \\
(1.62-1.71)\end{array}$ & $\begin{array}{l}1.97 \pm 0.22 \\
(1.92-2.01)\end{array}$ & $\begin{array}{l}1.56 \pm 0.27 \\
(1.50-1.63)\end{array}$ & 0.051 & $<0.05^{*}$ & $<0.05^{*}$ \\
\hline PPH VL & $\begin{array}{l}3.15 \pm 0.10 \\
(3.12-3.17)\end{array}$ & $\begin{array}{c}3 \pm 0.11 \\
(2.97-3.02)\end{array}$ & $\begin{array}{l}3.46 \pm 0.05 \\
(3.45-3.46)\end{array}$ & $\begin{array}{l}4.31 \pm 0.04 \\
(4.30-4.31)\end{array}$ & 0.411 & $<0.05^{*}$ & 0.873 \\
\hline PPH VR & $\begin{array}{l}1.56 \pm 0.33 \\
(1.48-1.63)\end{array}$ & $\begin{array}{l}1.26 \pm 0.27 \\
(1.20-1.31)\end{array}$ & $\begin{array}{l}1.26 \pm 0.22 \\
(1.21-1.30)\end{array}$ & $\begin{array}{l}1.68 \pm 0.29 \\
(1.61-1.74)\end{array}$ & $<0.001 * *$ & $<0.001 * *$ & $<0.05^{*}$ \\
\hline
\end{tabular}

Abbreviations: MTT ADD = first metatarsal bone in Adduction: I MTT toward to medial body line; MTT ABD = first metatarsal bone in Abduction: I MTT away to medial body line; MTT PF = first metatarsal bone in plantarflexión: I MTT toward to the floor; MTT DF = first metatarsal bone in dorsiflexión: I MTT away to the floor; MTT VL = first metatarsal bone spins to inside: I MTT toward medial body line; MTT VR = first metatarsal bone spin to outside: I MTT away to medial body line; UNSHOD = barefoot participants without any height in shoe heel; PPH ADD = proximal phalanx of hallux in Adduction: PPH toward to medial body line; PPH ABD = proximal phalanx of hallux in Abduction: PPH away to medial body line; PPH PF = proximal phalanx of hallux in plantarflexión: PPH toward to the floor; PPH DF = proximal phalanx of hallux in dorsiflexión: PPH away to the floor; PPH VL = proximal phalanx of hallux spins to inside: PPH toward medial body line; PPH VR = proximal phalanx of hallux spin to outside: PPH away to medial body line; \pm SD = standard deviation; $p$-value $<0.05 *$ (within a $95 \%$ confidence interval) was considered statistically significant; $p$-value $<0.001 * *$ (within a $95 \%$ confidence interval) was considered statistically strong significant; Movements and axes refer to the medial line of the body; all values are expressed in degrees.

Finally, Tables 6 and 7 summarize the Spearman's Rho correlation coefficients of static and dynamic conditions between the height of the heels and the movements of PPH and I MTT, respectively.

In static tests, adduction of IMTT had a statistically significant positive correlation while wearing $6 \mathrm{~cm}$ heels versus unshod condition $(0.5, p<0.001)$ as well as abduction $(0.347, p<0.001)$. Valgus values had a statistically significant positive correlation too under $3 \mathrm{~cm}$ high heel $(0.51, p<0.001)$. Regarding PPH abduction, there was a statistical positive correlation in $6 \mathrm{~cm}$ heels $(0.261, p<0.05)$ as well as a statistically significant negative correlation to adduction under $3 \mathrm{~cm}$ and $6 \mathrm{~cm}$ of high heels $(p<0.05)$.

During dynamic tests, $\mathrm{PPH}$ abduction had a statistically significant positive correlation with heels of $6 \mathrm{~cm}(0.527, p<0.001)$ combined with a statistically significant inverse correlation in adduction $(-0.278, p<0.05)$ with $6 \mathrm{~cm}$; there was a significant positive correlation with valgus values $(0.242$, $p<0.05)$. 
Table 6. STATIC Spearman-Rho correlation coefficients between variables.

\begin{tabular}{|c|c|c|c|c|c|c|}
\hline \multirow[t]{2}{*}{ Variable } & $\begin{array}{l}\text { Unshod vs. } \\
\text { Heel } 3 \mathrm{~cm}\end{array}$ & \multirow[t]{2}{*}{$p$-Value } & $\begin{array}{l}\text { Unshod vs. } \\
\text { Heel } 6 \mathrm{~cm}\end{array}$ & \multirow[t]{2}{*}{$p$-Value } & $\begin{array}{l}\text { Unshod vs. } \\
\text { Heel } 9 \mathrm{~cm}\end{array}$ & \multirow[t]{2}{*}{$p$-Value } \\
\hline & ( $\rho$-Coefficient) & & ( $\rho$-Coefficient) & & ( $\rho$-Coefficient) & \\
\hline MTT ADD & 0.256 & 0.3 & 0.5 & $<0.001^{* *}$ & 0.521 & $<0.001^{* *}$ \\
\hline MTT ABD & -0.69 & 0.77 & 0.347 & $<0.001^{* *}$ & 0.228 & $<0.05^{*}$ \\
\hline MTT PF & -0.189 & -0.192 & 0.723 & 0.061 & -0.125 & 0.75 \\
\hline MTT DF & 0.173 & 0.124 & -0.639 & 0.072 & 0.146 & 0.6 \\
\hline MTT VL & 0.510 & $<0.001 * *$ & -0.370 & 0.5 & 0.487 & 0.336 \\
\hline MTT VR & 0.26 & 0.122 & -0.401 & $<0.001^{* *}$ & 0.587 & 0.2 \\
\hline PPH ADD & 0.391 & 0.46 & 0.150 & -0.112 & 0.0984 & 0.226 \\
\hline PPH ABD & 0.412 & 0.477 & 0.261 & $<0.05^{*}$ & 0.58 & 0.398 \\
\hline PPH PF & 0.184 & 0.103 & -0.017 & 0.116 & -0.189 & 0.69 \\
\hline PPH DF & 0.054 & 0.111 & -0.079 & 0.086 & -0.141 & 0.333 \\
\hline PPH VL & 0.179 & -0.037 & 0.71 & 0.406 & 0.183 & 522 \\
\hline PPH VR & -0.306 & $<0.05^{*}$ & -0.222 & $<0.05^{*}$ & 0.36 & 0.922 \\
\hline
\end{tabular}

Abbreviations: $\rho$-coefficient $=$ Spearman Rho coefficient; MTT ADD $=$ first metatarsal bone in adduction: I MTT toward to medial body line; MTT ABD = first metatarsal bone in abduction: I MTT away to medial body line; MTT PF = first metatarsal bone in plantarflexion: I MTT toward to the floor; MTT DF = first metatarsal bone in dorsiflexion: I MTT away to the floor; MTT VL = first metatarsal bone spins to inside: I MTT toward medial body line; MTT VR = first metatarsal bone spin to outside: I MTT away to medial body line; UNSHOD = barefoot participants without any height in shoe heel; PPH ADD = proximal phalanx of hallux in adduction: PPH toward to medial body line; PPH ABD = proximal phalanx of hallux in abduction: $\mathrm{PPH}$ away to medial body line; $\mathrm{PPH}$ PF = proximal phalanx of hallux in plantarflexion: $\mathrm{PPH}$ toward to the floor; PPH DF = proximal phalanx of hallux in dorsiflexion: PPH away to the floor; PPH VL = proximal phalanx of hallux spins to inside: PPH toward medial body line; PPH VR = proximal phalanx of hallux spin to outside: $\mathrm{PPH}$ away to medial body line; $\pm \mathrm{SD}=$ standard deviation; $p$-value $<0.05$ * (within a 95\% confidence interval) was considered statistically significant; $p$-value < $0.001 * *$ (within a $95 \%$ confidence interval) was considered statistically strong significant; Movements and axes refer to the medial line of the body; all values are expressed in degrees.

Table 7. DINADYNAMIC Spearman-Rho correlation coefficients between variables.

\begin{tabular}{|c|c|c|c|c|c|c|}
\hline \multirow[t]{2}{*}{ Variable } & \multirow{2}{*}{$\begin{array}{c}\begin{array}{c}\text { Unshod vs. } \\
\text { Heel } 3 \mathrm{~cm}\end{array} \\
\text { ( } \rho \text {-Coefficient) }\end{array}$} & \multirow[t]{2}{*}{$p$-Value } & \multirow{2}{*}{$\begin{array}{c}\text { Unshod vs. } \\
\text { Heel } 6 \mathrm{~cm}\end{array}$} & \multirow[t]{2}{*}{$p$-Value } & \multirow{2}{*}{$\begin{array}{l}\text { Unshod vs. } \\
\text { Heel } 9 \mathrm{~cm}\end{array}$} & \multirow[t]{2}{*}{$p$-Value } \\
\hline & & & & & & \\
\hline MTT ADD & -0.276 & $<0.05^{*}$ & 0.025 & 0.061 & 0.181 & 0.3 \\
\hline MTT ABD & 0.306 & 0.189 & 0.606 & 0.138 & 0.258 & 0.224 \\
\hline MTT PF & 0.086 & -0.193 & 0.026 & -0.139 & -0.119 & 0.148 \\
\hline MTT DF & 0.056 & 0.203 & 0.043 & 0.128 & -0.142 & 0.956 \\
\hline MTT VL & 0.167 & 0.128 & -0.104 & 0.134 & 0.523 & $<0.001^{* *}$ \\
\hline MTT VR & -0.071 & 0.0733 & -0.088 & 0.0621 & 0.666 & 0.459 \\
\hline PPH ADD & -0.364 & $<0.001^{* *}$ & -0.278 & $<0.05^{*}$ & 0.856 & 0.1 \\
\hline PPH ABD & 0.71 & 0.25 & 0.527 & $<0.001^{* *}$ & 0.375 & 0.476 \\
\hline PPH PF & -0.128 & -0.005 & -0.028 & 0.148 & -0.109 & 0.945 \\
\hline PPH DF & 0.1 & -0.116 & 0.148 & 0.058 & 0.167 & 0.8 \\
\hline PPH VL & -0.169 & 0.150 & 0.242 & $<0.05^{*}$ & 0.154 & 0.36 \\
\hline PPH VR & -0.522 & $<0.001^{* *}$ & 0.108 & -0.089 & 0.146 & 0.221 \\
\hline
\end{tabular}

Abbreviations: $\rho$-coefficient $=$ Spearman Rho coefficient; MTT ADD = first metatarsal bone in Adduction: I MTT toward to medial body line; MTT ABD = first metatarsal bone in abduction: I MTT away to medial body line; MTT PF = first metatarsal bone in plantarflexion: I MTT toward to the floor; MTT DF = first metatarsal bone in dorsiflexion: I MTT away to the floor; MTT VL = first metatarsal bone spins to inside: I MTT toward medial body line; MTT VR = first metatarsal bone spin to outside: I MTT away to medial body line; UNSHOD = barefoot participants without any height in shoe heel; PPH ADD = proximal phalanx of hallux in adduction: PPH toward to medial body line; PPH ABD = proximal phalanx of hallux in abduction: $\mathrm{PPH}$ away to medial body line; $\mathrm{PPH} \mathrm{PF}=$ proximal phalanx of hallux in plantarflexion: $\mathrm{PPH}$ toward to the floor; PPH DF = proximal phalanx of hallux in dorsiflexion: PPH away to the floor; PPH VL = proximal phalanx of hallux spins to inside: PPH toward medial body line; PPH VR = proximal phalanx of hallux spin to outside: $\mathrm{PPH}$ away to medial body line; $\pm \mathrm{SD}=$ standard deviation; $p$-value $<0.05 *$ (within a 95\% confidence interval) was considered statistically significant; $p$-value < 0.001 ** (within a $95 \%$ confidence interval) was considered statistically strong significant; Movements and axes refer to the medial line of the body; all values are expressed in degrees. 


\section{Discussion}

HAV is a forefoot pathology related to PPH in valgus and abduction deviations plus the IMTT bone in adduction deviation $[1,2,51]$. Previous studies $[18,52-54]$ have shown the relationship between HAV development and the narrow toe tip footwear typical of the high heel shoes; other groups [1,27] speculated that high heels have an etiology factor in HAV development with only one prior review [12] considering the isolated high heels's effect on first MPJ deviation on its conclusions but without any further study. This research was the first study to use the Polhemus Fastrack ${ }^{\circledR}$ to assess the effects of high heels on static and dynamic conditions on PPH and IMTT. Thus, many statistically significant variables have been obtained, but of the 96 variables studied here across 80 subjects, only a few had statistically significant correlations. Due to the independent evaluation of the bones, we could not establish a direct discussion with the findings of other authors who interpreted HAV as a global forefoot disease [34]. In addition, this work has studied a healthy population without any limitation of mobility in their joints. Thus, a small difference in segment movements was expected.

Our results on the effect of high heels on PPH during dynamic testing showed that a high heel of $6 \mathrm{~cm}$ had a statistically significant increase in abduction in the transverse plane and valgus movement in the frontal plane through IMPJ. There was positive correlation of these values as well as a statistically significant reduction in the adduction; there was negative correlation to the barefoot condition. In addition, during static tests, there was a statistically significant abduction increase in PPH for high heels $6 \mathrm{~cm}$ or higher with a corresponding positive correlation; therefore, abduction is the only movement that appeared to have a positive correlation and statistical significance in both static and dynamic tests. Thus, we conclude that heels over $6 \mathrm{~cm}$ correlated with an abduction effect on PPH without the narrow shoe box interference. This agrees with arguments on the biomechanical development of HAV processes that claimed that the PPH was the first precursor bone segment to begin the HAV pathology [51,55-57] due to medial capsular tension ligaments that become hyper-elastic and let the PPH proceed to abduction deviation [7]. The PPH then has a strong push forward to the IMTT in the push off phase that can lead to adduction deviation.

More recently, Wang et al. [34] reported an increase in forefoot abduction while wearing $5 \mathrm{~cm}$ high-heeled shoes during walking vs. barefoot arguing that the squeezing effect of the high heels on the foot had a displacement toward the toe tip. This produced valgus and abduction of PPH. We agree with these conclusions, but we showed that the foot produces this "abductor effect" on PPH; it is not from the narrow box of the shoes. In addition, we obtained a large increase in the value of PPH abduction with a $3 \mathrm{~cm}$ heel. This result was not statistically significant.

The effects of high heels had contradictory effects on IMTT movements. There were no statistically significant results to justify its implication on HAV development in contrast with other groups that identified IMTT adduction and valgus [1,2] as well as inclination of the IMTT axis as risk factors of bunion [58] or IMCJ hypermobility [59]; both movements on transverse and frontal planes are under doubt because of a lack of objective data [60]. Surprisingly, it seemed that wearing any high heel might decrease valgus deviation of IMTT although this condition only had statistical significance with $6 \mathrm{~cm}$ heels; there was no positive correlation. The absence of concrete IMTT values related with typical HAV development suggests that PPH may be the principal bone to start the pathological process. This agrees with a study that identified the presence of HAV with greater reduction in size of the adductor hallucis muscle [61] as one of the most important muscles to balance the PPH.

In contrast to previous studies [62] that found no association between footwear characteristics (heel height and narrow box) and HAV development, our cohort had (18-38 years) had movement deviation of PPH in heels over $6 \mathrm{~cm}$. This agrees with other studies where older women reported HAV. They wore shoes with heels over $5 \mathrm{~cm}$ [12]. This work showed data on IMTT and PPH from the two different static and dynamic conditions and 3 kinds of high heels. Thus, we selected and summarized heel heights and determined that high heels could develop HAV. $6 \mathrm{~cm}$ was the common height for both static and dynamic situations; these different variables converged to induce HAV development. 
Most of the main limitations in other similar studies were equivocal results secondary to small sample size [19], differences in anthropometric characteristics of the subjects groups [63], or the inclusion of participants wearing their own high-heeled shoes [64]. This leads to a heterogeneous sample [19]. We studied a homogeneous sample that improved the measurement conditions of other groups that also failed to show ICC, CCC, SEM or MDC values [10,34]; our data had low to moderate correlation, and we considered these statistical parameters.

Coupling relationships between hindfoot inversion/eversion and forefoot abduction/adduction $\left(R^{2}=0.5\right)$ and hallux dorsiflexion/plantarflexion $\left(R^{2}=0.7\right)$ were the only prior references found on this topic [65]. It had similar Spearman values in that work but did show any contrast between data because the authors did not assess individual movements of any segment bone as PPH or I MTT, like in the present work. These are the only specific correlations done relative to PPH and IMTT in the literature and confirm the low Spearman correlations of our values.

\section{Limitations}

Bone segment measurements were performed with an electronic Polhemus Fastrack ${ }^{\circledR}$ goniometer and showed current instability due to inherent human gait fluctuations and sensor noise. The MDC values were higher than the grades obtained in the dynamic PPH valgus and abduction as well as in the static abduction PPH variables. Considering that the percent error set for the device was around $1.6 \%$-and considering that the \pm SD and SEM values obtained in the study were under this $1.6 \%$ - these results are considered to be statistically valid but with caution.

On the other hand, we were not able to study the effect of order on our sample because didn't write the different orders of each one neither the number of these selections. We assumed the possible "order effect" as "perfectly balanced" because all the study subgroups of each station have the same number of subjects and this can dilutes the "order effect". Future study design should include a section of studying the effect of order of experiments.

\section{Conclusions}

Wearing shoes with heels over $6 \mathrm{~cm}$ may produce a valgus and abduction increase in PPH movement. This abduction is specifically detected in the development of HAV pathology. Future cohort studies will be required to clarify the time period that is needed to develop HAV pathology related to high-heeled shoes.

Author Contributions: Conceptualization, R.S.-G. and R.B.d.B.-V.; Methodology, R.S.-G. and R.B.d.B.-V.; Software, E.M.M.-J.; Validation, C.C.-L. and C.R.-M.; Formal Analysis, C.C.-L.; Investigation, M.E.L.-I.; Resources, P.P.-L. and D.L.-L.; Data Curation, P.P.-L. and D.L.-L.; Writing-Original Draft Preparation, R.S.-G.; Writing-Review \& Editing, R.S.-G., R.B.d.B.-V., C.C.-L., C.R.-M., E.M.M.-J., M.E.L.-I., P.P.-L. and D.L.-L.; Visualization, D.L.-L. and E.M.M.-J.; Supervision, P.P.-L. and M.E.L.-I.; Project Administration, C.C.-L. and C.R.-M.; Funding Acquisition, anyone.

Funding: This research received no specific grant from any funding agency in the public, commercial, or not-for-profit sectors.

Conflicts of Interest: The authors declare no conflict of interest.

\section{References}

1. Mann, R.A.; Coughlin, M.J. Hallux valgus-Etiology, anatomy, treatment and surgical considerations. Clin. Orthop. Relat. Res. 1981, 157, 31-41. [CrossRef]

2. Piqué-Vidal, C.V.J. A geometric analysis of hallux valgus: Correlation with clinical assessment of severity. J. Foot Ankle Res. 2009, 2, 15. [CrossRef] [PubMed]

3. Menz, H.B.; Munteanu, S.E. Radiographic validation of the Manchester scale for the classification of hallux valgus deformity. Rheumatology 2005, 44, 1061-1066. [CrossRef] [PubMed]

4. Wolff, J. The classic: On the inner architecture of bones and its importance for bone growth. 1870. Clin. Orthop. Relat. Res. 2010, 468, 1056-1065. [CrossRef] [PubMed] 
5. Root, M.L.; Orien, W.P.W.H. Normal and Abnormal Function of the Foot; Corp, C.B.: Los Angeles, CA, USA, 1977; Volume II.

6. McBride, I.D.; Wyss, U.P.; Cooke, T.D.; Murphy, L.; Phillips, J.; Olney, S.J. First metatarsophalangeal joint reaction forces during high-heel gait. Foot Ankle 1991, 11, 282-288. [CrossRef] [PubMed]

7. Hicks, J. The mechanics of the foot. II. The plantar aponeurosis and the arch. J. Anat. 1954, 88, 25-30. [PubMed]

8. Faber, F.W.; Kleinrensink, G.J.; Mulder, P.G.; Verhaar, J.A. Mobility of the first tarsometatarsal joint in hallux valgus patients: A radiographic analysis. Foot Ankle Int. 2001, 22, 965-969. [CrossRef] [PubMed]

9. Nix, S.; Smith, M.; Vicenzino, B. Prevalence of hallux valgus in the general population: A systematic review and meta-analysis. J. Foot Ankle Res. 2010, 3, 21. [CrossRef]

10. Kawakami, W.; Takahashi, M.; Iwamoto, Y.; Shinakoda, K. Coordination Among Shank, Rearfoot, Midfoot, and Forefoot Kinematic Movement During Gait in Individuals with Hallux Valgus. J. Appl. Biomech. 2018, 35, 44-51. [CrossRef]

11. Coughlin, M.J.; Jones, C.P. Hallux Valgus: Demographics, Etiology, and Radiographic Assessment. Foot Ankle Int. 2007, 28, 759-777. [CrossRef]

12. Nguyen, U.S.; Hillstrom, H.J.; Li, W.; Dufour, A.B.; Kiel, D.P.; Procter-Gray, E.; Gagnon, M.M.; Hannan, M.T. Factors associated with hallux valgus in a population-based study of older women and men: The MOBILIZE Boston Study. Osteoarthr. Cartil. 2010, 18, 41-46. [CrossRef] [PubMed]

13. Owoeye, B.A.; Akinbo, S.R.; Aiyegbusi, A.L.; Ogunsola, M.O. Prevalence of hallux valgus among youth population in Lagos, Nigeria. Niger. Postgrad. Med. J. 2011, 18, 51-55. [PubMed]

14. Dawson, J.; Thorogood, M.; Marks, S.A.; Juszczak, E.; Dodd, C.; Lavis, G.; Fitzpatrick, R. The prevalence of foot problems in older women: A cause for concern. J. Public Health Med. 2002, 24, 77-84. [CrossRef] [PubMed]

15. Menz, H.B.; Lord, S.R. Foot problems, functional impairment, and falls in older people. J. Am. Podiatr. Med. Assoc. 1999, 89, 458-467. [CrossRef] [PubMed]

16. Glassy, C.M.; Glassy, M.S.; Guggenheim, C. Relationship between self-reported high-heeled shoe use and bone mineral density using quantitative ultrasound at a community health fair. Clin. Rheumatol. 2013, 32, 37-41. [CrossRef] [PubMed]

17. Gerber, S.B.; Costa, R.V.; Grecco, L.A.C.; Pasini, H.; Marconi, N.F.; Oliveira, C.S. Interference of high-heeled shoes in static balance among young women. Hum. Mov. Sci. 2012, 31, 1247-1252. [CrossRef] [PubMed]

18. Snow, R.E.; Williams, K.R. High heeled shoes: Their effect on center of mass position, posture, three-dimensional kinematics, rearfoot motion, and ground reaction forces. Arch. Phys. Med. Rehabil. 1994, 75, 568-576.

19. Cronin, N.J. The effects of high heeled shoes on female gait: A review. J. Electromyogr. Kinesiol. 2014, 24, 258-263. [CrossRef]

20. Mika, A.; Oleksy, Ł.; Kielnar, R.; Świerczek, M. The influence of high- and low-heeled shoes on balance in young women. Acta Bioeng. Biomech. 2016, 18, 97-103.

21. Barnish, M.S.; Barnish, J. High-heeled shoes and musculoskeletal injuries: A narrative systematic review. BMJ Open 2016, 6, e010053. [CrossRef]

22. Hong, W.-H.; Lee, Y.-H.; Lin, Y.-H.; Tang, S.F.T.; Chen, H.-C. Effect of shoe heel height and total-contact insert on muscle loading and foot stability while walking. Foot Ankle Int. 2013, 34, 273-281. [CrossRef] [PubMed]

23. Nyska, M.; McCabe, C.; Linge, K.; Klenerman, L. Plantar foot pressures during treadmill walking with high-heel and low-heel shoes. Foot Ankle Int. 1996, 17, 662-666. [CrossRef] [PubMed]

24. Yung-Hui, L.; Wei-Hsien, H. Effects of shoe inserts and heel height on foot pressure, impact force, and perceived comfort during walking. Appl. Ergon. 2005, 36, 355-362. [CrossRef]

25. Penny, J.Ø.; Speedtsberg, M.B.; Kallemose, T.; Bencke, J. Can an off-the-rack orthotic stiletto alter pressure and comfort scores in the forefoot, arch and heel? Ergonomics 2018, 61, 1130-1138. [CrossRef] [PubMed]

26. Dufour, A.B.; Casey, V.A.; Golightly, Y.M.; Hannan, M.T. Characteristics associated with hallux valgus in a population-based foot study of older adults. Arthritis Care Res. 2014, 66, 1880-1886. [CrossRef] [PubMed]

27. Wülker, N.; Mittag, F. The treatment of hallux valgus. Dtsch. Arztebl. Int. 2012, 109, 857-867. [CrossRef] [PubMed]

28. Mann, R.A.; Coughlin, M.J. Surgery of the Foot and Ankle, 6th ed.; Mosby: St Louis, MO, USA, 1992. 
29. Swanson, J.E.; Stoltman, M.G.; Oyen, C.R.; Mohrbacher, J.A.; Orandi, A.; Olson, J.M.; Glasoe, W.M. Comparison of 2D-3D Measurements of Hallux and First Ray Sagittal Motion in Patients with and Without Hallux Valgus. Foot Ankle Int. 2016, 37, 227-232. [CrossRef] [PubMed]

30. Deschamps, K.; Birch, I.; Desloovere, K.; Matricali, G.A. The impact of hallux valgus on foot kinematics: A cross-sectional, comparative study. Gait Posture 2010, 32, 102-106. [CrossRef]

31. Gur, G.; Ozkal, O.; Dilek, B.; Aksoy, S.; Bek, N.; Yakut, Y. Effects of Corrective Taping on Balance and Gait in Patients with Hallux Valgus. Foot Ankle Int. 2017, 38, 532-540. [CrossRef]

32. Klugarova, J.; Janura, M.; Svoboda, Z.; Sos, Z.; Stergiou, N.; Klugar, M. Hallux valgus surgery affects kinematic parameters during gait. Clin. Biomech. 2016, 40, 20-26. [CrossRef]

33. Nawoczenski, D.A.; Ludewig, P.M. The Effect of Forefoot and Arch Posting Orthotic Designs on First Metatarsophalangeal Joint Kinematics During Gait. J. Orthop. Sport Phys. Ther. 2004, 34, 317-327. [CrossRef]

34. Wang, M.; Gu, Y.; Baker, J.S. Analysis of foot kinematics wearing high heels using the Oxford foot model. Technol. Health Care 2018, 26, 815-823. [CrossRef]

35. Becerro de Bengoa Vallejo, R.; Gomez, R.S.; Losa Iglesias, M.E. Clinical improvement in functional hallux limitus using a cut-out orthosis. Prosthet. Orthot. Int. 2016, 40, 215-223. [CrossRef]

36. Hong, W.-H.; Lee, Y.-H.; Chen, H.-C.; Pei, Y.-C.; Wu, C.-Y. Influence of heel height and shoe insert on comfort perception and biomechanical performance of young female adults during walking. Foot Ankle Int. 2005, 26, 1042-1048. [CrossRef]

37. Buell, T.; Green, D.R.; Risser, J. Measurement of the first metatarsophalangeal joint range of motion. J. Am. Podiatr. Med. Assoc. 1988, 78, 439-448. [CrossRef]

38. Umberger, B.R.; Nawoczenski, D.A.; Baumhauer, J.F. Reliability and validity of first metatarsophalangeal joint orientation measured with an electromagnetic tracking device. Clin. Biomech. 1999, 14, 74-76. [CrossRef]

39. Halstead, J.; Redmond, A.C. Weight-bearing passive dorsiflexion of the hallux in standing is not related to hallux dorsiflexion during walking. J. Orthop. Sports Phys. Ther. 2006, 36, 550-556. [CrossRef]

40. Du, W.-Y.; Huang, T.-S.; Hsu, K.-C.; Lin, J.-J. Measurement of scapular medial border and inferior angle prominence using a novel scapulometer: A reliability and validity study. Musculoskelet. Sci. Pract. 2017, 32, 120-126. [CrossRef]

41. Nawoczenski, D.A.; Cook, T.M.; Saltzman, C.L. The Effect of Foot Orthotics on Three-Dimensional Kinematics of the Leg and Rearfoot During Running. J. Orthop. Sport Phys. Ther. 1995, 21, 317-327. [CrossRef]

42. Milne, A.D.; Chess, D.G.; Johnson, J.A.; King, G.J. Accuracy of an electromagnetic tracking device: A study of the optimal range and metal interference. J. Biomech. 1996, 29, 791-793. [CrossRef]

43. Welsh, B.J.; Redmond, A.C.; Chockalingam, N.; Keenan, A.-M. A case-series study to explore the efficacy of foot orthoses in treating first metatarsophalangeal joint pain. J. Foot Ankle Res. 2010, 3, 17. [CrossRef] [PubMed]

44. Shereff, M.J.; Bejjani, F.J.; Kummer, F.J. Kinematics of the first metatarsophalangeal joint. J. Bone Jt. Surg. Am. 1986, 68, 392-398. [CrossRef]

45. Nawoczenski, D.A.; Baumhauer, J.F.; Umberger, B.R. Relationship between clinical measurements and motion of the first metatarsophalangeal joint during gait. J. Bone Jt. Surg. Am. 1999, 81, 370-376. [CrossRef]

46. Ramanathan, A.K.; John, M.C.; Arnold, G.P.; Cochrane, L.; Abboud, R.J. The effects of off-the-shelf in-shoe heel inserts on forefoot plantar pressure. Gait Posture 2008, 28, 533-537. [CrossRef] [PubMed]

47. Landis, J.R.; Koch, G.G. The measurement of observer agreement for categorical data. Biometrics 1977, 33, 159-174. [CrossRef] [PubMed]

48. Li, L. A concordance correlation coefficient to evaluate reproducibility. Biometrics 1989, 45, 255-268.

49. Jacobson, N.; Truax, P. Clinical significance: A statistical approach to defining meaningful change in psychotherapy research. J. Consult. Clin. Psychol. 1991, 59, 12-19. [CrossRef] [PubMed]

50. Portney, L.; Watkins, M. Foundations of Clinical Research: Applications to Practice, 3rd ed.; Hall PP: Upper Saddle River, NJ, USA, 2009.

51. Eustace, S.; O’Byrne, J.; Stack, J.; Stephens, M.M. Radiographic features that enable assessment of first metatarsal rotation: The role of pronation in hallux valgus. Skelet. Radiol. 1993, 22, 153-156. [CrossRef]

52. Frey, C. Foot health and shoewear for women. Clin. Orthop. Relat. Res. 2000, 372, 32-44. [CrossRef]

53. Ashizawa, K.; Kumakura, C.; Kusumoto, A.; Narasaki, S. Relative foot size and shape to general body size in Javanese, Filipinas and Japanese with special reference to habitual footwear types. Ann. Hum. Biol. 1997, 24, 117-129. [CrossRef] 
54. Menz, H.B.; Morris, M.E. Footwear Characteristics and Foot Problems in Older People. Gerontology 2005, 51, 346-351. [CrossRef] [PubMed]

55. Saltzman, C.L.; Aper, R.L.; Brown, T.D. Anatomic determinants of first metatarsophalangeal flexion moments in hallux valgus. Clin. Orthop. Relat. Res. 1997, 339, 261-269. [CrossRef]

56. Arinci İncel, N.; Genç, H.; Erdem, H.R.; Yorgancioglu, Z.R. Muscle Imbalance in Hallux Valgus. Am. J. Phys. Med. Rehabil. 2003, 82, 345-349. [CrossRef]

57. Shereff, M.J. Pathophysiology, anatomy, and biomechanics of hallux valgus. Orthopedics 1990, 13, 939-945. [PubMed]

58. Glasoe, W.M.; Jensen, D.D.; Kampa, B.B.; Karg, L.K.; Krych, A.R.; Pena, F.A.; Ludewig, P.M. First Ray Kinematics in Women with Rheumatoid Arthritis and Bunion Deformity: A Gait Simulation Imaging Study. Arthritis Care Res. 2014, 66, 837-843. [CrossRef] [PubMed]

59. Lapidus, P.W. A quarter of a century of experience with the operative correction of the metatarsus varus primus in hallux valgus. Bull. Hosp. Jt. Dis. 1956, 17, 404-421.

60. Doty, J.F.; Coughlin, M.J. Hallux valgus and hypermobility of the first ray: Facts and fiction. Int. Orthop. 2013, 37, 1655-1660. [CrossRef]

61. Aiyer, A.; Stewart, S.; Rome, K. The effect of age on muscle characteristics of the abductor hallucis in people with hallux valgus: A cross-sectional observational study. J. Foot Ankle Res. 2015, 8, 19. [CrossRef]

62. Menz, H.B.; Roddy, E.; Marshall, M.; Thomas, M.J.; Rathod, T.; Peat, G.M.; Croft, P.R. Epidemiology of Shoe Wearing Patterns Over Time in Older Women: Associations with Foot Pain and Hallux Valgus. J. Gerontol. Ser. A Biol. Sci. Med. Sci. 2016, 71, 1682-1687. [CrossRef]

63. Cronin, N.J.; Barrett, R.S.; Carty, C.P. Long-term use of high-heeled shoes alters the neuromechanics of human walking. J. Appl. Physiol. 2012, 112, 1054-1058. [CrossRef]

64. Csapo, R.; Maganaris, C.N.; Seynnes, O.R.; Narici, M.V. On muscle, tendon and high heels. J. Exp. Biol. 2010, 213 Pt 15, 2582-2588. [CrossRef]

65. Dubbeldam, R.; Nester, C.; Nene, A.V.; Hermens, H.J.; Buurke, J.H. Kinematic coupling relationships exist between non-adjacent segments of the foot and ankle of healthy subjects. Gait Posture 2013, 37, 159-164. [CrossRef] [PubMed] 